\title{
A Reasoning System of Ternary Projective Relations
}

\author{
Eliseo Clementini, Spiros Skiadopoulos, Roland Billen, Francesco Tarquini
}

\begin{abstract}
This paper introduces a reasoning system based on a previously developed model for ternary projective relations between spatial objects. The model applies to spatial objects of the kind point and region, is based on basic projective invariants and takes into account the size and shape of the three objects that are involved in a relation. The reasoning system proposes a set of permutation and composition rules, which allow the inference of unknown relations from given ones.
\end{abstract}

\section{INTRODUCTION}

The field of Qualitative Spatial Reasoning (QSR) has experienced a great interest in the spatial data handling community due to its potential applications [1]. An important topic in QSR is the definition of reasoning systems on qualitative spatial relations. For example, regarding topological relations, the 9-intersection model [2] provides formal definitions for the relations and a reasoning system based on composition tables [3] establishes a mechanism to find new relations from a set of given ones.

As discussed in [4], geometric properties can be subdivided in three groups: topological, projective and metric. Most qualitative relations between spatial objects can be defined in terms of topological or projective properties [5]. Qualitative distances are a qualitative interpretation of metric distances [6].

Projective relations are a category of spatial relations that can be described by projective properties of the space without resorting to metric properties [7]. Projective relations are thus qualitative in nature because they do not need exact measures to be explained. Projective relations are more specific than topological relations and can serve as a basis for describing relations that are not captured by topology. At an intermediate rank between metrics and topology, projective relations are as much varied as "right of", "before", "between", "along", "surrounded by", "in front of", "back", "north of", "east of", and so on.

To have a common sense understanding of projective relations, it is helpful to think about different two-dimensional views of a three-dimensional real world scene of objects: changing the point of view, metric aspects such distances and angles among the objects appear to be different, but there are properties that are common in all the views. These common properties are projective properties.

Likewise topological relations, which are defined by using the connectedness topological invariant, projective relations

E. Clementini is with the Dept. of Electrical and Information Engineering, University of L'Aquila, Italy. Email: eliseo@ing.univaq.it

S. Skiadopoulos is with the Dept. of Computer Science and Technology, University of Peloponnese, Hellas. Email: spiros@uop.gr

R. Billen is with the Dept. of Geography, University of Liege, Belgium. Email: rbillen@ulg.ac.be

F. Tarquini is with the Dept of Electrical and Information Engineering, University of L'Aquila, Italy. Email: tarquini@ing.univaq.it can be defined by using the collinearity projective invariant, which is the property of three collinear points being still collinear after an arbitrary number of projections. A main difference in the treatment of topological relations and projective relations is that, while basic topological relations are binary, basic projective relations are ternary because they are defined on the collinearity of three points. The definition of collinearity has been extended to regions in [8].

In this paper, we propose a reasoning system for the set of projective relations that was introduced in [9]. Such relations establish a jointly exhaustive and pairwise disjoint (JEPD) set of projective relations among any three regions of the plane. A preliminary version of this model was presented in [10], but the set of relations was not JEPD. A first version of the reasoning system, applied on a subset of the relations, was presented in [11]. The projective relations are ternary relations of the kind $r(A, B, C)$, where $A$ has the role of primary object and $B$ and $C$ have the role of reference objects. This latter terminology derives from the work on positional relations (e.g., [7]), where the position of an object (primary) is stated with respect to the position of one or more other objects acting as reference. Two cases can be distinguished based on whether the convex hulls of the reference objects are disjoint or not disjoint. In the first case, the model, called the 5-intersection, by using only projective concepts partitions the plane into five acceptance areas with respect to the reference objects; in the second case, the partition of the plane results in two acceptance areas. The model is able to differentiate between 34 different projective relations that are obtained by computing the intersection of the primary object with the acceptance areas that are determined by the reference objects.

The reasoning system establishes rules of permutation and composition of relations in the form of tables. Among the 34 projective relations of the model, we can distinguish singletile and multi-tile relations, depending whether the primary object intersects one or more of the acceptance areas. The single-tile relations are five for non-intersecting convex hulls of reference objects and two for intersecting convex hulls of reference objects. The permutation rules are of two types: converse and rotation. Regarding the composition table, we initially find it for the composition of single-tile relations with all basic relations (therefore, a $7 \times 34$ table). The latter table has been found in two different ways: by manually checking all geometric configurations that satisfy the table and by running a simulation program with a high number of random regions and finding the occurring relations. Afterwards, the full composition table has been found $(34 \times 34)$ with algebraic rules that can be applied to the $7 \times 34$ table.

The remainder of the paper is organized as follows. We start in Section II with a brief comparison to existing literature. In Section III, we introduce preliminaries on reasoning with 
ternary relations. In Sections IV, we summarize the model of projective relations among points and, in Section $V$, we describe the reasoning system for points. In Section VI, we summarize the model for projective relations among regions. Section VII presents the main contribution of the paper, consisting in the reasoning system for regions. Section VIII draws conclusions. In the appendix, we include all geometric configurations that have been considered to build the composition tables.

\section{RELATED WORK}

In the QSR literature, we can find various models for reasoning with projective relations. Freksa's double-cross calculus [12] is similar to our approach in the case of points. Such a calculus, as it has been further discussed in [13], [14], is based on ternary directional relations between points. In Freksa's model, an intrinsic frame of reference centred in a given point partitions the plane into four quadrants that are given by the front-back and right-left dichotomies. This leads to a greater number of qualitative distinctions with different algebraic properties and composition tables. A smaller number of qualitative distinctions and an independence from the specific frame of reference would improve the possibility of extending this model to other spatial types besides points.

Other work on ternary calculi is rather limited. Most approaches consider binary relations to which a frame of reference is associated [15], [16]. Exceptions of ternary relations, such as "between", were considered in [17] and, more recently, in [18]. Projective relations, intended as locative expressions between two objects [19], depend on an underlying frame of reference. The use of ternary relations instead of binary ones allows us to describe the projective relation in a way that is independent from the frame of reference. The ternary model of projective relations can be seen as a geometric abstraction of locative expressions commonly used in the physical world.

Our approach can be compared to various models for orientation relations [20], [21], [22], [23], [24] or cardinal directions between points [25], [26]. Most of them, even when explicitly related to projective geometry, never avoid the use of metric properties (minimum bounding rectangles, angles, etc.) and external frames of reference (such as a grid). To this respect, the main difference in our approach is that we only deal with projective invariants, independently of metric aspects such as distances and angles.

Most work on cardinal directions deals with point abstractions of spatial features and less work has been devoted to extended objects [27], [28], [29], [30], [31]. In [32], the authors use spheres surrounding the objects to take into account the shape of objects in relative orientation. In [27], the authors develop a model for cardinal directions between extended objects, where the partition of the plane is determined by the prolongations of the sides of the minimum bounding rectangle (MBR) of a reference object. The reasoning for such a model has been developed in [33], [34]. Contrary to the double-cross calculus [12] and the direction matrix [27], we use a cone-based model instead of a projection-based model [25].
Ternary projective relations are more general than binary orientation relations or cardinal directions, because they don't need to refer to an external frame of reference. The two reference objects are able to make a partition of the plane to which the position of the primary object is compared. In [35], we find the same approach as ours regarding the reasoning on points, except that authors consider a partition of the plane in seven parts instead of five. Moving from points to extended regions, among the strengths of our model are the facts that it uses projective properties only and that the acceptance areas of relations depend on the shape and size of the reference objects. Independence from a specific frame of reference (both egocentric or allocentric [36]) allows us to specify ternary relations among objects from a purely geometric point of view. A further step is to apply the model of ternary projective relations to specific settings, like robot navigation [35], [37] or cardinal directions on the Earth surface [38].

\section{REASONING WITH RELATIONS}

Most research on spatial and temporal relations have focused on binary relations and studied algorithms for several useful operators like converse and composition [39], [3], [25], [40], [33]. The converse of a relation $r$ that holds between $A$ and $B$, denoted by $r^{\smile}$, specifies the relation between $B$ and $A$. The composition of a relation $r$ between $A$ and $B$ with a relation $q$ between $B$ and $C$, denoted by $r \circ q$, specifies the relation between $A$ and $C$. Converse and composition are used to construct reasoning mechanisms applicable to query processing.

The aforementioned notions can also be extended for ternary relations [14], [41], [13]. Let us consider a set of basic ternary relations $\mathcal{T}$ that contains $|\mathcal{T}|$ jointly exhaustive and pairwise disjoint relations [16]. Elements of $\mathcal{T}$ are used to represent definite information. Using these relations, we can define the powerset of $\mathcal{T}$ (i.e., the set of all subsets), denoted by $2^{\mathcal{T}}$ that contains $2^{|\mathcal{T}|}$ relations. Elements of $2^{\mathcal{T}}$ can be used to represent definite but also indefinite information. For instance, if $t_{1}, \ldots, t_{n}$ are basic relations in $\mathcal{T}$, then $\left\{t_{1}, \ldots, t_{n}\right\}$ is a relation in $2^{\mathcal{T}}$ which is equivalent to $t_{1} \vee \cdots \vee t_{n}$.

Initially, we define converse and rotation. If $r(A, B, C)$ holds, the converse of $r$ specifies the relation between $A, C$ and $B$ while the rotation of $r$ specifies the relation between $C, A$ and $B$

Definition 1: Let $r$ be a ternary relation in $\mathcal{T}$. The converse of $r$, denoted by $r^{\smile}$, is a relation in $2^{\mathcal{T}}$ defined as:

$$
r^{\smile}=\{t \in \mathcal{T} \mid(\exists A, B, C)(t(A, C, B) \wedge r(A, B, C))\}
$$

Definition 2: Let $r$ be a ternary relation in $\mathcal{T}$. The rotation of $r$, denoted by $r \frown$, is a relation in $2^{\mathcal{T}}$ defined as:

$$
r \frown=\{t \in \mathcal{T} \mid(\exists A, B, C)(t(C, A, B) \wedge r(A, B, C))\}
$$

Notice that the converse and rotation operators as defined above are the ternary counterpart of the binary converse operation [41]. For binary relations, the converse operation is sufficient since there are only 2 permutations between 2 objects $A$ and $B$, namely $(A, B)$ and $(B, A)$. For the ternary relations case, there are 6 possible permutations between 3 
objects $A, B$ and $C$, namely $(A, B, C),(A, C, B),(B, A, C)$, $(B, C, A),(C, A, B)$ and $(C, B, A)$. It is easy to verify that we need both converse and rotation to move between these permutations. For instance, we may move from $(A, B, C)$ to $(C, B, A)$ by applying the rotation followed by the converse operator.

Next, we define ternary composition.

Definition 3: Let $r$ and $q$ be two ternary relations. The composition of $r$ and $q$, denoted by $r \circ q$, is defined as:

$$
\begin{gathered}
r \circ q=\{t \in \mathcal{T} \mid(\exists A, B, C, D)(r(A, B, C) \wedge q(B, C, D) \wedge \\
t(A, C, D))\}
\end{gathered}
$$

Similarly to earlier works in qualitative spatial relations, we use a weak definition of converse and composition [42], [43], [33], [31]. Typically, these operators are expressible for every pair of spatial relations and can be naturally used as a constraint propagation mechanism. On the contrary, the more strict set-theoretic definitions of converse and composition are not always definable [40], [44], [33], [31].

In the following sections, we will define a model for ternary projective relations for points and a model for ternary projective relations for regions. Moreover, we will study the converse, the rotation and the composition operations for the above models.

\section{Projective Relations FOR POINTS}

Our basic set of projective relations for points is based on the most important geometric invariant in a projective space: the collinearity of three points. Therefore, the nature of projective relations is intrinsically ternary. Let us consider three points $P_{1}, P_{2}$ and $P_{3}$. To define the projective relation $r_{1}\left(P_{1}, P_{2}, P_{3}\right)$ of primary point $P_{1}$ with respect to reference points $P_{2}$ and $P_{3}$, we consider the following two cases.

Case 1: $P_{2} \neq P_{3}$. In this case, to define $r_{1}\left(P_{1}, P_{2}, P_{3}\right)$, we use the directed line $\overrightarrow{P_{2} P_{3}}$. This directed line partitions the space into 5 parts that correspond to the relations rightside, leftside, before, after and between and are denoted by rightside $\left(P_{2}, P_{3}\right)$, leftside $\left(P_{2}, P_{3}\right)$, before $\left(P_{2}, P_{3}\right)$, $\operatorname{after}\left(P_{2}, P_{3}\right)$ and between $\left(P_{2}, P_{3}\right)$, respectively (Figure 1a). In Figure $1 \mathrm{a}$, rightside $\left(P_{2}, P_{3}\right)$ is the light gray area, leftside $\left(P_{2}, P_{3}\right)$ is the dark gray area, before $\left(P_{2}, P_{3}\right)$ is the dotted semi-line, after $\left(P_{2}, P_{3}\right)$ is the dashed semi-line and between $\left(P_{2}, P_{3}\right)$ is the thick line segment. Notice that:

- All parts are disjoint.

- The union of all parts is $\Re^{2}$.

- $P_{2} \in \operatorname{between}\left(P_{2}, P_{3}\right), P_{3} \in \operatorname{between}\left(P_{2}, P_{3}\right), \overrightarrow{P_{2} P_{3}} \notin$ rightside $\left(P_{2}, P_{3}\right)$ and $\overrightarrow{P_{2} P_{3}} \notin$ leftside $\left(P_{2}, P_{3}\right)$.

If a point $P_{1}$ is included (in the set-theoretic sense) in region rightside $\left(P_{2}, P_{3}\right)$ of some points $P_{2}$ and $P_{3}$, then we say that $P_{1}$ is rightside of $P_{2}$ and $P_{3}$ and we write $\operatorname{rs}\left(P_{1}, P_{2}, P_{3}\right)$. Similarly, we can define relations $l s$ (leftside), bf (before), af (after) and bt (between).

Example 1: For the points of Figure 1b, we have:

$$
r s\left(P_{1}, P_{2}, P_{3}\right) \text {, af }\left(P_{A}, P_{2}, P_{3}\right) \text { and } b t\left(P_{2}, P_{2}, P_{3}\right) .
$$

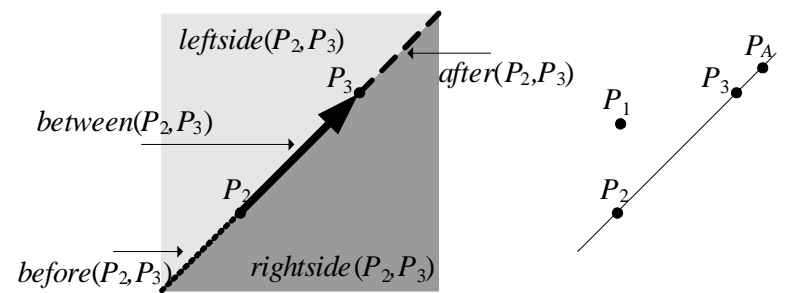

(a)

(b)

Fig. 1. Ternary relations between points $\left(P_{2} \neq P_{3}\right)$

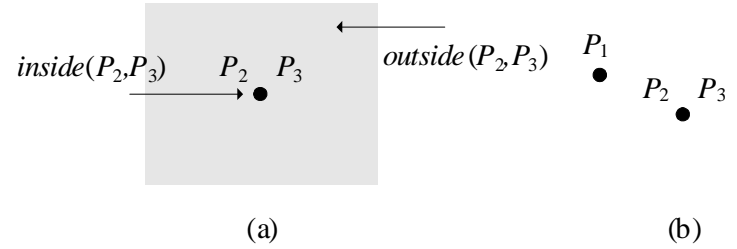

Fig. 2. Ternary relations between points $\left(P_{2}=P_{3}\right)$

Case 2: $P_{2}=P_{3}$. In this case, the space is partitioned in a point $\left(P_{2}\right)$ and an open area $\left(\Re^{2}-P_{2}\right)$. These regions correspond to the relations inside and outside and are denoted by inside $\left(P_{2}, P_{3}\right)$ and outside $\left(P_{2}, P_{3}\right)$ respectively (Figure 2a).

If a point $P_{1}$ is included (in the set-theoretic sense) in region outside $\left(P_{2}, P_{3}\right)$ of some points $P_{2}$ and $P_{3}$, then we say that $P_{1}$ is outside of $P_{2}$ and $P_{3}$ and we write ou $\left(P_{1}, P_{2}, P_{3}\right)$. Similarly, we can define relation inside, denoted by $i n$.

Example 2: For the points of Figure 2b, we have:

$$
\text { ou }\left(P_{1}, P_{2}, P_{3}\right) \text { and } \operatorname{in}\left(P_{2}, P_{2}, P_{3}\right) .
$$

Summarizing, the set of projective relations between points contains the following $7(=5+2)$ relations: $r s, l s, b f, a f, b t$, in and ou. We will use $\mathcal{D}_{\text {point }}$ to denote this set. Relations in $\mathcal{D}_{\text {point }}$ are jointly exhaustive and pairwise disjoint [9]. Elements of $\mathcal{D}_{\text {point }}$ can be used to represent definite information, e.g., $b t\left(P_{1}, P_{2}, P_{3}\right)$. To express indefinite information, we use the powerset $2^{\mathcal{D}_{\text {point }}}$, of $\mathcal{D}_{\text {point }}$ which contains $128=2^{7}$ relations. For instance, $\{b t$, af $\}\left(P_{1}, P_{2}, P_{3}\right) \in 2^{\mathcal{D}_{\text {point }}}$ denotes that point $P_{1}$ is either between or after points $P_{2}$ and $P_{3}$ (i.e.,bt $\left(P_{1}, P_{2}, P_{3}\right) \vee a f\left(P_{1}, P_{2}, P_{3}\right)$ holds). More details about these relations can be found in [9].

\section{REASONING WITH PROJECTIVE RELATIONS FOR POINTS}

In this section, we will study the converse, rotation and composition operators for the projective relations for points defined in Section IV.

For any projective relation $r\left(P_{1}, P_{2}, P_{3}\right)$, Table I presents the relations that correspond to the converse $r^{\smile}\left(P_{1}, P_{3}, P_{2}\right)$ and the rotation $r \frown\left(P_{3}, P_{1}, P_{2}\right)$ of $r\left(P_{1}, P_{2}, P_{3}\right)$. For example, given three points $P_{1}, P_{2}$ and $P_{3}$ such that $b f\left(P_{1}, P_{2}, P_{3}\right)$ holds, using Table I, we can derive that $a f\left(P_{1}, P_{3}, P_{2}\right)$ and af $\left(P_{3}, P_{1}, P_{2}\right)$ also hold (see also Figure $3 a$ ). 


\begin{tabular}{|c||c|c|}
\hline$r\left(P_{1}, P_{2}, P_{3}\right)$ & $r^{\smile}\left(P_{1}, P_{3}, P_{2}\right)$ & $r^{\frown}\left(P_{3}, P_{1}, P_{2}\right)$ \\
\hline \hline$b t$ & $b t$ & $b t, b f, o u$ \\
\hline$r s$ & $l s$ & $r s$ \\
\hline$b f$ & $a f$ & $a f$ \\
\hline$l s$ & $r s$ & $l s$ \\
\hline$a f$ & $b f$ & $b t$ \\
\hline$i n$ & $i n$ & $i n$ \\
\hline$o u$ & $o u$ & $b t$ \\
\hline \multicolumn{2}{|c}{} \\
\hline
\end{tabular}

TABLE I

CONVERSE AND ROTATION FOR PROJECTIVE RELATIONS BETWEEN POINTS

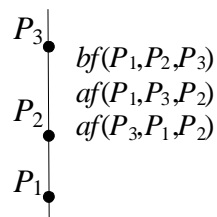

(a)

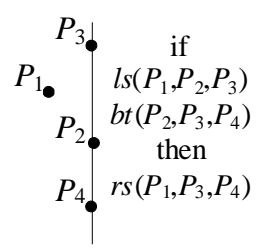

(b)

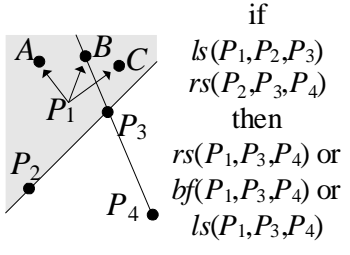

(c)
Fig. 3. Operations on point relations

Table II illustrates the result of the composition, $r_{1} \circ$ $r_{2}\left(P_{1}, P_{3}, P_{4}\right)$, of two basic projective relations $r_{1}\left(P_{1}, P_{2}, P_{3}\right)$ and $r_{2}\left(P_{2}, P_{3}, P_{4}\right)$. For instance, as we can verify in Figure $3 \mathrm{~b}$, we have:

$$
l s\left(P_{1}, P_{2}, P_{3}\right) \circ b t\left(P_{2}, P_{3}, P_{4}\right)=r s\left(P_{1}, P_{3}, P_{4}\right) .
$$

Notice that, for some cases, the result of the composition is a relation in $2^{\mathcal{D}_{\text {point }}}$. For example, we have:

$$
l s\left(P_{1}, P_{2}, P_{3}\right) \circ r s\left(P_{2}, P_{3}, P_{4}\right)=\{r s, b f, l s\}\left(P_{1}, P_{3}, P_{4}\right) .
$$

This means that, given four points $P_{1}, P_{2}, P_{3}$ and $P_{4}$ such that $l s\left(P_{1}, P_{2}, P_{3}\right)$ and $r s\left(P_{2}, P_{3}, P_{4}\right)$ hold, the projective relation of $P_{1}$ can be rightside or before or leftside $P_{3}$ and $P_{4}$ (i.e., $\left.r s\left(P_{1}, P_{3}, P_{4}\right) \vee b f\left(P_{1}, P_{3}, P_{4}\right) \vee l s\left(P_{1}, P_{3}, P_{4}\right)\right)$. This can be verified in Figure 3c. If $P_{1}$ were placed in point $A$ (respectively $B$ and $C$ ) then the first (respectively the second and the third) disjunct would be verified.

Moreover, for some other cases the result is impossible, denoted by $I M P$. For instance, in $\left(P_{1}, P_{2}, P_{3}\right) \circ$ $r s\left(P_{2}, P_{3}, P_{4}\right)=I M P$ because we can easily verify that there do not exist points $P_{1}, P_{2}, P_{3}$ and $P_{4}$ such that in $\left(P_{1}, P_{2}, P_{3}\right)$ and $\operatorname{rs}\left(P_{2}, P_{3}, P_{4}\right)$ simultaneously hold.

\section{Projective Relations For REgions}

In Sections IV and V, we have defined a model for projective relations for points and we have studied reasoning operators. We will now turn our attention to regions. In this paper, we will consider regions that are formed by finite unions of regions that are homeomorphic to the closed unit disk [34]. This set of regions is denoted by $R E G^{*}$. Regions in $R E G^{*}$ are regular closed point sets and can be disconnected or have holes. However, class $R E G^{*}$ excludes points, lines and regions with emanating lines. Let $A$ be a region in $R E G^{*}$, we denote the convex hull of $A$ by $C H(A)$.
In this section, we will briefly present the projective model for regions, we refer the interested reader to [9] for a more extended discussion. These relations extend the projective relations for points discussed in Section IV, thus they are also ternary. Let us consider three regions $R_{1}, R_{2}$ and $R_{3}$. To define the projective relation $r_{1}\left(R_{1}, R_{2}, R_{3}\right)$ of primary region $R_{1}$ with respect to reference regions $R_{2}$ and $R_{3}$, we consider the following two cases.

Case 1: $C H\left(R_{2}\right) \cap C H\left(R_{3}\right)=\emptyset$. In this case, to define $r_{1}\left(R_{1}, R_{2}, R_{3}\right)$, we use the convex hull of the union of regions $R_{2}$ and $R_{3}$ and the internal common tangents of regions $R_{2}$ and $R_{3}$. Since $C H\left(R_{2}\right) \cap C H\left(R_{3}\right)=\emptyset$ holds, we can always define exactly two internal and two external common tangents. For instance, in Figure 4a, we illustrate two regions $\left(R_{2}\right.$ and $R_{3}$ ) and their internal and external common tangents.

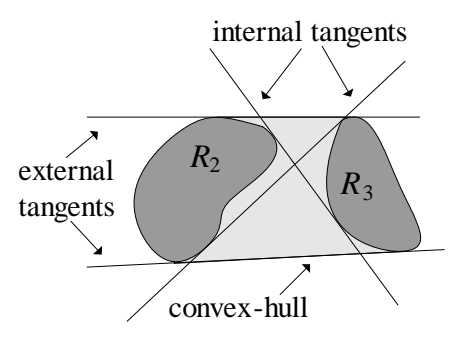

(a)

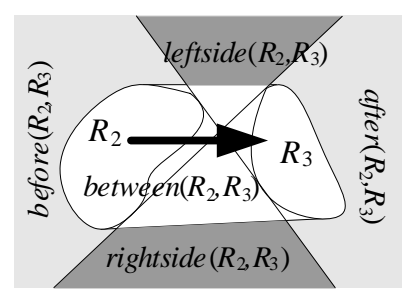

(b)
Fig. 4. Ternary relations between regions $\left(C H\left(R_{2}\right) \cap C H\left(R_{3}\right)=\emptyset\right)$

The external common tangents help to find the convex hull of the union of $R_{2}$ and $R_{3}$. The convex hull and the internal common tangents of regions $R_{2}$ and $R_{3}$ partition the reference space into 5 regions as in (Figure $4 \mathrm{~b}$ ). Formal definitions of these regions can be found in [9]. Similarly to the point case, these areas correspond to the relations rightside, leftside, before, after and between and are denoted by rightside $\left(P_{2}, P_{3}\right)$, leftside $\left(P_{2}, P_{3}\right)$, before $\left(P_{2}, P_{3}\right)$, $\operatorname{after}\left(P_{2}, P_{3}\right)$ and between $\left(P_{2}, P_{3}\right)$ respectively (Figure $4 \mathrm{~b}$ ). To distinguish the above areas, we consider an oriented line from the first reference region (i.e., $R_{2}$ ) to the second reference region (i.e., $\left.R_{3}\right)$. Specifically, in Figure $4 \mathrm{~b}$, rightside $\left(P_{2}, P_{3}\right)$ is the lower dark gray area, leftside $\left(P_{2}, P_{3}\right)$ is the upper dark gray area, before $\left(P_{2}, P_{3}\right)$ is the light gray area on the left, $\operatorname{after}\left(P_{2}, P_{3}\right)$ is the light gray area on the right and between $\left(P_{2}, P_{3}\right)$ is the white area on the middle. Notice that:

- The union of all regions is $\Re^{2}$.

- All areas but between $\left(R_{2}, R_{3}\right)$ are unbounded.

- Area between $\left(R_{2}, R_{3}\right)$ is closed.

- The interiors of all areas are disjoint but two areas may share common points in their boundaries. For instance, the areas before $\left(R_{2}, R_{3}\right)$ and rightside $\left(R_{2}, R_{3}\right)$ share some points of the internal tangent.

Even though tiles share some points in their borders there is no ambiguity in defining projective relations because the class $R E G^{*}$ does not contain objects that could lie entirely on the borderline (like lines and points). 


\begin{tabular}{|c||c|c|c|c|c|c|c|}
\hline$r_{2} / r_{1}$ & $b t$ & $r s$ & $b f$ & $l s$ & $a f$ & in & ou \\
\hline \hline$b t$ & $b t$ & $l s$ & $\{b t, a f\}$ & $r s$ & $b f$ & $b t$ & $\{b t, r s, b f, l s, a f\}$ \\
\hline$r s$ & $r s$ & $\{b t, r s, l s, a f\}$ & $r s$ & $\{r s, b f, l s\}$ & $l s$ & $I M P$ & $I M P$ \\
\hline$b f$ & $b f$ & $r s$ & $b f$ & $l s$ & $\{b t, a f\}$ & $I M P$ & $I M P$ \\
\hline$l s$ & $l s$ & $\{r s, b f, l s\}$ & $l s$ & $\{b t, r s, l s, a f\}$ & $r s$ & $I M P$ & $I M P$ \\
\hline$a f$ & $\{b t, a f\}$ & $l s$ & $a f$ & $r s$ & $b f$ & $I M P$ & $I M P$ \\
\hline$i n$ & $I M P$ & $I M P$ & $I M P$ & $I M P$ & $I M P$ & $i n$ & in \\
\hline ou & $o u$ & $i n, o u$ & $o u$ & $o u$ & $o u$ & $I M P$ & $I M P$ \\
\hline
\end{tabular}

TABLE II

COMPOSITION TABLE OF PROJECTIVE RELATIONS BETWEEN POINTS

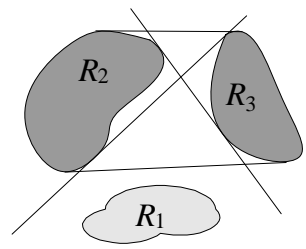

(a)

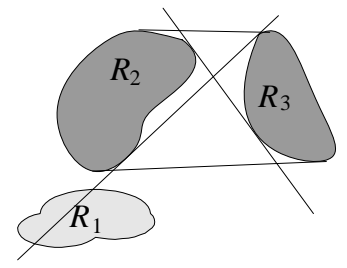

(b)
Fig. 5. Example of projective relations between regions $\left(\mathrm{CH}\left(\mathrm{R}_{2}\right) \cap\right.$ $\left.C H\left(R_{3}\right)=\emptyset\right)$

If a region $R_{1}$ is included (in the set-theoretic sense) in region rightside $\left(R_{2}, R_{3}\right)$ of some reference regions $R_{2}$ and $R_{3}$ (Figure $5 \mathrm{a}$ ), then we say that $R_{1}$ is rightside of $R_{2}$ and $R_{3}$ and we write $r s\left(R_{1}, R_{2}, R_{3}\right)$. Similarly, we can define relations $l s$ (leftside), bf (before), af (after) and bt (between).

If a primary region $R_{1}$ lies partly in the rightside $\left(R_{2}, R_{3}\right)$ area and partly in the before $\left(R_{2}, R_{3}\right)$ area of some reference regions $R_{2}$ and $R_{3}$ (Figure $5 \mathrm{~b}$ ), then we say that $R_{1}$ is partly rightside and partly before of $R_{2}$ and $R_{3}$ and we write $r s: b f\left(R_{1}, R_{2}, R_{3}\right)$.

Case 2: $C H\left(R_{2}\right) \cap C H\left(R_{3}\right) \neq \emptyset$. In this case, the common internal tangents of regions $R_{2}$ and $R_{3}$ cannot be defined. Thus, we only use the convex hull of regions $R_{2}$ and $R_{3}$ to partition the reference space into two areas as in Figure 6a. These areas correspond to relations inside and outside and are denoted by inside $\left(R_{2}, R_{3}\right)$ and outside $\left(R_{2}, R_{3}\right)$ respectively.

Region inside $\left(R_{2}, R_{3}\right)$ is bounded while outside $\left(R_{2}, R_{3}\right)$ is unbounded. Similarly to Case 1 , the union of regions inside $\left(R_{2}, R_{3}\right)$ and outside $\left(R_{2}, R_{3}\right)$ is $\Re^{2}$ and the interiors of these areas are disjoint but they share common points in their boundaries.

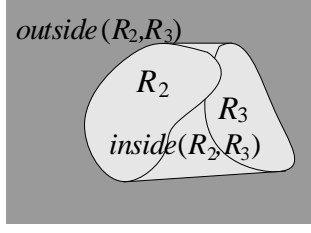

(a)

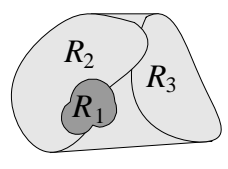

(b)

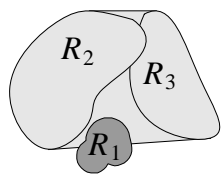

(c)
Fig. 6. Ternary relations between points $\left(C H\left(R_{2}\right) \cap C H\left(R_{3}\right) \neq \emptyset\right)$ region inside $\left(R_{2}, R_{3}\right)$ of some reference regions $R_{2}$ and $R_{3}$ (Figure 6b), then we say that $R_{1}$ is inside of $R_{2}$ and $R_{3}$ and we write $i n\left(R_{1}, R_{2}, R_{3}\right)$. Similarly, we can define relation ou (outside).

If a primary region $R_{1}$ lies partly in the inside $\left(R_{2}, R_{3}\right)$ area and partly in the outside $\left(R_{2}, R_{3}\right)$ area of some reference regions $R_{2}$ and $R_{3}$ (Figure 6c) then we say that $A$ is partly inside and partly outside of $R_{2}$ and $R_{3}$ and we write in:ou $\left(R_{1}, R_{2}, R_{3}\right)$.

Summarizing, the general definition of a basic projective relation in our framework is given as follows:

Definition 4: A basic projective relation is an expression $r_{1}: \cdots: r_{k}$ where $k$ and $r_{1}, \ldots, r_{k}$ can belong to exactly one of the following cases.

1) $1 \leq k \leq 5, r_{1}, \ldots, r_{k} \in\{b t, r s, b f, l s, a f\}$ and $R_{i} \neq$ $R_{j}$ for every $i, j$ such that $1 \leq i, j \leq k$ and $i \neq j$.

2) $1 \leq k \leq 2, r_{1}, \ldots, r_{k} \in\{$ in, ou $\}$ and $R_{i} \neq R_{j}$ for every $i, j$ such that $1 \leq i, j \leq k$ and $i \neq j$.

We refer to $r_{1}, \ldots, r_{k}$ as the tiles of relation $r_{1}: \cdots: r_{k}$. Moreover, a basic projective relation $r_{1}: \cdots: r_{k}$ is called singletile if $k=1$; otherwise it is called multi-tile.

In order to avoid confusion, we will write the elements of a multi-tile relation according to the following order: $b t, r s$, $b f, l s, a f$, in and $o u$. Thus, we always write $r s: b f$ and not $b f: r s$.

Example 3: Expressions $r s, r s: b f$, in and in:ou are basic projective relations. The first and the third are single-tile relations, while the second and the forth are multi-tile. Objects involved in these relations are shown in Figures $5 \mathrm{a}, 5 \mathrm{~b}, 6 \mathrm{~b}$ and $6 \mathrm{c}$ respectively.

The following two definitions formally define basic (single and multi-tile) projective relations.

Definition 5: Let $R_{1}, R_{2}$ and $R_{3}$ be three regions in $R E G^{*}$.

If a region $R_{1}$ is included (in the set-theoretic sense) in 
Then, single-tile projective relations are defined as follows.

$$
\begin{array}{lcl}
b t\left(R_{1}, R_{2}, R_{3}\right) \quad \text { iff } & R_{1} \in \text { between }\left(R_{2}, R_{3}\right) \\
& \text { and } & C H\left(R_{2}\right) \cap C H\left(R_{3}\right)=\emptyset \\
r s\left(R_{1}, R_{2}, R_{3}\right) \quad \text { iff } & R_{1} \in \text { rightside }\left(R_{2}, R_{3}\right) \\
& \text { and } & C H\left(R_{2}\right) \cap C H\left(R_{3}\right)=\emptyset \\
b f\left(R_{1}, R_{2}, R_{3}\right) \quad \text { iff } & R_{1} \in \text { before }\left(R_{2}, R_{3}\right) \\
& \text { and } & C H\left(R_{2}\right) \cap C H\left(R_{3}\right)=\emptyset \\
l s\left(R_{1}, R_{2}, R_{3}\right) \quad \text { iff } & R_{1} \in \text { leftside }\left(R_{2}, R_{3}\right) \\
& \text { and } & C H\left(R_{2}\right) \cap C H\left(R_{3}\right)=\emptyset \\
a f\left(R_{1}, R_{2}, R_{3}\right) \quad \text { iff } & R_{1} \in \text { after }\left(R_{2}, R_{3}\right) \\
& \text { and } & C H\left(R_{2}\right) \cap C H\left(R_{3}\right)=\emptyset \\
i n\left(R_{1}, R_{2}, R_{3}\right) & \text { iff } & R_{1} \in \text { inside }\left(R_{2}, R_{3}\right) \\
& \text { and } & C H\left(R_{2}\right) \cap C H\left(R_{3}\right) \neq \emptyset \\
\text { ou }\left(R_{1}, R_{2}, R_{3}\right) \quad \text { iff } & R_{1} \in \text { outside }\left(R_{2}, R_{3}\right) \\
& \text { and } & C H\left(R_{2}\right) \cap C H\left(R_{3}\right) \neq \emptyset
\end{array}
$$

Definition 6: Let $R_{1}, R_{2}$ and $R_{3}$ be three regions in $R E G^{*}$ and $r_{1}: \cdots: r_{k}$ be a multi-tile projective relation. Then, $r_{1}: \cdots: r_{k}\left(R_{1}, R_{2}, R_{3}\right)$ holds iff there exist regions $Q_{1}, \ldots, Q_{k} \in R E G^{*}$ such that $r_{1}\left(Q_{1}, R_{2}, R_{3}\right), \ldots, r_{k}\left(Q_{k}, R_{2}, R_{3}\right)$ and $R_{1}=Q_{1} \cup \cdots \cup Q_{k}$.

Summarizing, the set of basic projective relations between regions contains 34 relations (7 single-tile and 27 multi-tile). We will use $\mathcal{D}_{\text {region }}$ to denote this set. Relations in $\mathcal{D}_{\text {region }}$ are jointly exhaustive and pairwise disjoint [9]. Elements of $\mathcal{D}_{\text {region }}$ can be used to represent definite information about directional information. We will use $r, q$ and $p$ possibly subscripted to denote variables ranging in $\mathcal{D}_{\text {region }}$. To also

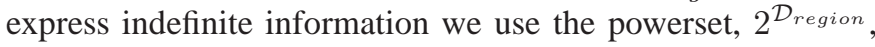
of $\mathcal{D}_{\text {region. }}$. We will use $\bar{r}, \bar{q}$ and $\bar{p}$ possibly subscripted to denote variables ranging in $2^{\mathcal{D}_{\text {region }}}$

\section{REASONING WITH PROJECTIVE RELATIONS FOR REGIONS}

To present our results, we will need the following definitions.

Definition 7: Let $r_{1}, \ldots, r_{k}$ be single-tile relations. The $\delta$ combination of relations $r_{1}, \ldots, r_{k}$, denoted by $\delta\left(r_{1}, \ldots, r_{k}\right)$, is a short-cut for the projective relation in $2^{\mathcal{D}_{\text {region }}}$ that can be constructed by combining single-tile relations $r_{1}, \ldots, r_{k}$.

For instance, we have $\delta(b t, r s, l s)=\{b t, r s, l s, b t: r s$, $b t: l s, r s: l s, b t: r s: l s\}$.

Definition 8: Let $r_{1}: \cdots: r_{k}$ be a basic projective relation in $\mathcal{D}_{\text {region }}$. The $\delta$-combination of $r_{1}: \cdots: r_{k}$ is defined as:

$$
\delta\left(r_{1}: \cdots: r_{k}\right)=\delta\left(r_{1}, \ldots, r_{k}\right)
$$

For instance, we have $\delta(b t: r s: l s)=\delta(b t, r s, l s)=$ $\{b t, r s, l s, b t: r s, b t: l s, r s: l s, b t: r s: l s\}$.

Definition 9: Let $\bar{r}_{1}=\left\{r_{1}^{1}, \ldots, r_{1}^{k_{1}}\right\}, \ldots, \bar{r}_{m}=$ $\left\{r_{m}^{1}, \ldots, r_{m}^{k_{m}}\right\}$ be $m$ projective relations in $2^{\mathcal{D}_{\text {region }}}$ (where $r_{1}^{1}, \ldots, r_{1}^{k_{1}}, \ldots, r_{m}^{1}, \ldots, r_{m}^{k_{m}}$ are basic projective relations). The $\delta$-combination of these relations is defined as:

$$
\delta\left(\bar{r}_{1}, \ldots, \bar{r}_{m}\right)=\delta\left(r_{1}^{1}, \ldots, r_{1}^{k_{1}}, \ldots, r_{m}^{1}, \ldots, r_{m}^{k_{m}}\right)
$$

Finally, we define

$$
\begin{gathered}
\delta\left(\delta\left(r_{1}^{1}, \ldots, r_{1}^{k_{1}}\right), \ldots, \delta\left(r_{m}^{1}, \ldots, r_{m}^{k_{m}}\right)\right)= \\
\delta\left(r_{1}^{1}, \ldots, r_{1}^{k_{1}}, \ldots, r_{m}^{1}, \ldots, r_{m}^{k_{m}}\right)
\end{gathered}
$$

Example 4: We have $\delta(\{b t, r s\}, \delta(r s, l s))=\delta(b t, r s, l s)=$ $\{b t, r s, l s, b t: r s, b t: l s, r s: l s, b t: r s: l s\}$.

Definition 10: We denote by $U_{\text {proj }}$ the universal disjunctive projective relation, i.e.,

$$
U_{\text {proj }}=\delta(b t, r s, b f, l s, a f, i n, o u) .
$$

Moreover, we denote by $D_{d}$ the universal disjunctive projective relation with reference regions with disjoint convex hulls, i.e., $D_{d}=\delta(b t, r s, b f, l s, a f)$, and by $D_{c}$ the universal disjunctive projective relation with reference regions with nondisjoint convex hulls, i.e., $D_{c}=\delta(i n, o u)$.

Definition 11: The tile-union of basic relations $r_{1}, \ldots, r_{k}$, denoted by tile-union $\left(r_{1}, \ldots, r_{k}\right)$, is the basic relation that consists of all the tiles of relations $r_{1}, \ldots, r_{k}$.

Example 5: We have tile-union $(r s, b f: l s)=r s: b f: l s$ and tile-union (bt, rs, rs:ls) = bt:rs:ls.

Definition 12: Let $\bar{r}_{1}=\left\{r_{1}^{1}, \ldots, r_{1}^{k_{1}}\right\}, \ldots, \bar{r}_{m}=$ $\left\{r_{m}^{1}, \ldots, r_{m}^{k_{m}}\right\}$ be $m$ projective relations in $2^{\mathcal{D}_{\text {region }}}$. The product of relations $\bar{r}_{1}, \ldots, \bar{r}_{m}$, denoted by $\pi\left(\bar{r}_{1}, \ldots, \bar{r}_{m}\right)$ is a projective relation in $2^{\mathcal{D}_{\text {region }}}$ defined as:

$$
\begin{gathered}
\pi\left(\bar{r}_{1}, \ldots, \bar{r}_{m}\right)=\left\{\text { tile-union }\left(s_{1}, \ldots, s_{m}\right) \mid\right. \\
\left.s_{1} \in\left\{r_{1}^{1}, \ldots, r_{1}^{k_{1}}\right\}, \ldots, s_{m} \in\left\{r_{m}^{1}, \ldots, r_{m}^{k_{m}}\right\}\right\} .
\end{gathered}
$$

Example 6: The following are some examples of products.

- $\pi(\{b t, r s\},\{b f, l s\})=\{b t: b f, b t: l s, r s: b f, r s: l s\}$,

- $\pi(\{r s: l s, b t\},\{b t: r s: l s, r s: b f: l s\})=\{b t: r s: l s, r s: b f: l s$, $b t: r s: b f: l s\}$

- $\pi(\{r s: l s, b t\},\{b t: r s: l s, r s: b f: l s\}, b f)=\{r s: b f: l s$, $b t: r s: b f: l s\}$.

The following is a useful proposition.

Proposition 1: Let $\bar{r}_{1}, \bar{r}_{2}$ and $\bar{q}$ be projective relations in $2^{\mathcal{D}_{\text {region }}}$. Then

$$
\pi\left(\bar{r}_{1} \cup \bar{r}_{2}, \bar{q}\right)=\pi\left(\bar{r}_{1}, \bar{q}\right) \cup \pi\left(\bar{r}_{2}, \bar{q}\right) .
$$

The product of relations is commonly used to compute the composition operator for other models of qualitative direction relations [33], [31]. In our work, we also need the following definition that uses the product of two relations.

Definition 13: The augmentation of a basic projective relation $r$ in $\mathcal{D}_{\text {region }}$ by a projective relation $\bar{q}$ in $2^{\mathcal{D}_{\text {region }}}$, denoted by $r / \bar{q}$ is a projective relation in $2^{\mathcal{D}_{\text {region }}}$ defined as:

$$
r / \bar{q}=r \cup \pi(r, \bar{q}) .
$$

Example 7: We have $r s: l s / \delta(b t, b f)=\{r s: l s, b t: r s: l s$, $r s: b f: l s, b t: r s: b f: l s\}$.

\section{A. Converse and rotation}

For any basic projective relation $r\left(R_{1}, R_{2}, R_{3}\right)$, Table III presents the relations that correspond to the converse $r^{\smile}\left(R_{1}, R_{3}, R_{2}\right)$ and the rotation $r \frown\left(R_{3}, R_{1}, R_{2}\right)$ of $r\left(R_{1}, R_{2}, R_{3}\right)$.

Example 8: Using Table III, we have that $r s^{\smile}=l s$ and $r s \frown=\{r s, o u\}$. These equations can be verified using regions $R_{1}, R_{2}$ and $R_{3}$ of Figure 7 . We have $l s \in r_{s}$ since in Figure $7 \mathrm{a}$ and $\mathrm{b}, \operatorname{rs}\left(R_{1}, R_{2}, R_{3}\right), \operatorname{ls}\left(R_{1}, R_{3}, R_{2}\right)$ holds. Similarly, we have (a) rs $\in$ rs $\frown$ since in Figure 7a, 


\begin{tabular}{|c|c|c|}
\hline$r\left(R_{1}, R_{2}, R_{3}\right)$ & $r \smile\left(R_{1}, R_{3}, R_{2}\right)$ & $r \frown\left(R_{3}, R_{1}, R_{2}\right)$ \\
\hline$\overline{b t}$ & $\overline{b t}$ & $\begin{array}{l}r s: l s / \delta(b t, b f) \cup b t / \delta(r s, l s) \cup \delta(b t, b f) \cup b f: a f / \delta(b t, l s) \cup b f: a f / \delta(b t, r s) \cup \\
b f: l s / b t \cup r s: b f / b t \cup b t: a f / l s \cup b t: a f / r s \cup D_{c}\end{array}$ \\
\hline$r s$ & ls & $\{r s, o u\}$ \\
\hline$b f$ & $a f$ & $a f / \delta(r s, l s) \cup r s: l s \cup o u$ \\
\hline ls & rs & $\{l s, o u\}$ \\
\hline$a f$ & $b f$ & $b t / \delta(r s, b f, l s) \cup r s: l s / b f$ \\
\hline bt:rs & $b t: l s$ & $\delta(b t, b f) / r s \cup b t / \delta(r s, b f, a f) \cup b f: a f / r s \cup D_{c}$ \\
\hline$b t: b f$ & $b t: a f$ & $b t / \delta(r s, b f, a f) \cup b f: a f / r s \cup b t / \delta(b f, l s, a f) \cup b f: a f / l s \cup D_{c}$ \\
\hline$b t: l s$ & bt:rs & $\delta(b t, b f) / l s \cup b t / \delta(b f, l s, a f) \cup b f: a f / l s \cup D_{c}$ \\
\hline$\overline{b t: a f}$ & $b t: b f$ & $\begin{array}{l}b t / \delta(r s, b f, l s) \cup r s: l s / b f \cup b f / b t \cup b f: a f / \delta(b t, l s) \cup b f: a f / \delta(b t, r s) \cup b f: l s / b t \cup \\
r s: b f / b t \cup b t: a f / l s \cup b t: a f / r s \cup D_{c}\end{array}$ \\
\hline$r s: b f$ & ls:af & $a f / r s \cup o u$ \\
\hline rs:ls & rs:ls & $a f \cup \delta(b t, b f) \cup D_{c}$ \\
\hline$r s: a f$ & $b f: l s$ & $b t / \delta(r s, b f) \cup r s: b f \cup\{i n, i n: o u\}$ \\
\hline$b f: l s$ & $r s: a f$ & af $/ l s \cup o u$ \\
\hline$b f: a f$ & $b f: a f$ & $b t / \delta(r s, b f, a f) \cup b f: a f / r s \cup b t / \delta(b f, l s, a f) \cup b f: a f / l s \cup D_{c}$ \\
\hline$l s: a f$ & $r s: b f$ & $b t / \delta(b f, l s) \cup b f: l s \cup\{i n, i n: o u\}$ \\
\hline$b t: r s: b f$ & $b t: l s: a f$ & $b t / \delta(r s, b f, a f) \cup b f: a f / r s \cup D_{c}$ \\
\hline$b t: r s: l s$ & bt:rs:ls & $\delta(b t, b f) \cup D_{c}$ \\
\hline$b t: r s: a f$ & $b t: b f: l s$ & $\delta(b t, b f) \cup r s: b f \cup b t / \delta(r s, b f, a f) \cup b f: a f / r s \cup D_{c}$ \\
\hline$b t: b f: l s$ & bt:rs:af & $b t / \delta(b f, l s, a f) \cup b f: a f / l s \cup D_{c}$ \\
\hline$b t: b f: a f$ & $b t: b f: a f$ & $b t / \delta(r s, b f, a f) \cup b f: a f / r s \cup b t / \delta(b f, l s, a f) \cup b f: a f / l s \cup D_{c}$ \\
\hline$b t: l s: a f$ & $b t: r s: b f$ & $\delta(b t, b f) \cup b f: l s \cup b t / \delta(b f, l s, a f) \cup b f: a f / l s \cup D_{c}$ \\
\hline$r s: b f: l s$ & $r s: l s: a f$ & $a f \cup D_{c}$ \\
\hline$r s: b f: a f$ & $b f: l s: a f$ & $b t / \delta(r s, b f, a f) \cup b f: a f / r s \cup D_{c}$ \\
\hline rs:ls:af & $r s: b f: l s$ & $b t / b f \cup i n \cup i n: o u$ \\
\hline$b f: l s: a f$ & $r s: b f: a f$ & $b t / \delta(b f, l s, a f) \cup b f: a f / l s \cup D_{c}$ \\
\hline$b t: r s: b f: l s$ & $b t: r s: l s: a f$ & $D_{c}$ \\
\hline$b t: r s: b f: a f$ & $b t: b f: l s: a f$ & $b t / \delta(r s, b f, a f) \cup b f: a f / r s \cup D_{c}$ \\
\hline bt:rs:ls:af & $b t: r s: b f: l s$ & $b t / b f \cup i n \cup i n: o u$ \\
\hline$b t: b f: l s: a f$ & $b t: r s: b f: a f$ & $b t / \delta(b f, l s, a f) \cup b f: a f / l s \cup D_{c}$ \\
\hline$r s: b f: l s: a f$ & $r s: b f: l s: a f$ & $\{i n, i n: o u\}$ \\
\hline$b t: r s: b f: l s: a f$ & $b t: r s: b f: l s: a f$ & $\{$ in, in:ou $\}$ \\
\hline$i n$ & in & $b t / D_{d} \cup r s: l s / D_{d} \cup b f: a f / D_{d} \cup D_{c}$ \\
\hline ou & ou & $b t / \delta(r s, l s, a f) \cup r s / \delta(b t, l s, a f) \cup l s / \delta(b t, r s, a f) \cup a f / \delta(b t, r s, l s) \cup D_{c}$ \\
\hline in:ou & in:ou & $b t / D_{d} \cup r s: l s / D_{d} \cup b f: a f / D_{d} \cup D_{c}$ \\
\hline
\end{tabular}

TABLE III

CONVERSE AND ROTATION FOR PROJECTIVE RELATIONS BETWEEN REGIONS

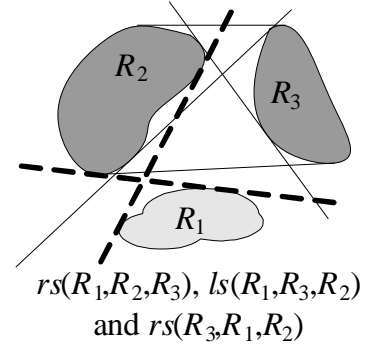

(a)

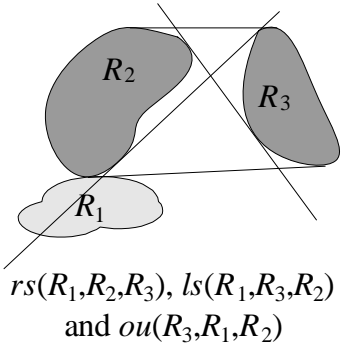

(b)
Fig. 7. Converse and rotation example

$r s\left(R_{1}, R_{2}, R_{3}\right)$ and $r s\left(R_{2}, R_{3}, R_{1}\right)$ and $(b)$ ou $\in r s \frown$ since in Figure $7 \mathrm{~b}, r s\left(R_{1}, R_{2}, R_{3}\right)$ and ou $\left(R_{3}, R_{1}, R_{2}\right)$ holds.

To compute the converse relations presented in Table III, we use the following proposition.

Proposition 2: The converse $r^{\smile}\left(R_{1}, R_{3}, R_{2}\right)$ of a relation $r\left(R_{1}, R_{2}, R_{3}\right)$ can be computed by performing the following substitutions to the tiles of $r$.

\begin{tabular}{|c|c|}
\hline Tiles of $r$ & Replace with (to get $r^{\smile}$ ) \\
\hline \hline$b t$ & $b t$ \\
\hline$r s$ & $l s$ \\
\hline$l s$ & $r s$ \\
\hline$b f$ & $a f$ \\
\hline$a f$ & $b f$ \\
\hline
\end{tabular}

Proof: The proof easily follows from the symmetry of the projective relations.

For instance, the converse of relation $r s$ is relation $l s$ and the converse of relation bt:rs:bf is relation bt:ls:af (see also Table III).

The rotation operation is much more involved. To compute the rotation operator, we have implemented Algorithm CoMPUTERotation (Figure 8). Given three regions $R_{1}, R_{2}$ and $R_{3}$, the above algorithm computes relation $r\left(R_{1}, R_{2}, R_{3}\right)$ and its rotation $q\left(R_{3}, R_{1}, R_{2}\right)$ (Step 4$)$. Algorithms for computing projective relations were presented in [9]. Regions $R_{1}, R_{2}$ and $R_{3}$ are constructed by the union of two rectangles (Step 3) that are taken from a large array of random rectangles $\mathcal{R}$ (Step 1). We consider regions formed by the union of two rectangles because simple rectangles are not general enough to satisfy 
multi-tile relations such as $b f: a f$ or $r s: l s$. In our experiments, we have varied the size of array $\mathcal{R}$ from 100,000 to $1,000,000$.

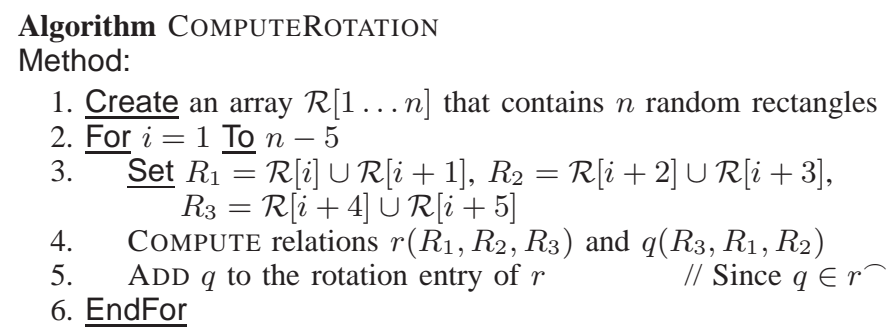

Fig. 8. Algorithm ComputeRotation

The results that Algorithm COMPUTERotATION produces are definitely sound since for every result there is an actual configuration of regions satisfying the relation and its rotation. In fact, besides running the algorithm, we checked the results of the table by exhaustively drawing all the configurations, making sure that no results are missing. With another routine, we then manipulate the output of the table to find the compact form with the $\delta$ operator. The final result is presented in Table III.

\section{B. Composing a single-tile with a basic relation}

For any single-tile relation $r_{1}(A, B, C)$ and any basic (single or multi-tile) relation $r_{2}(B, C, D)$, Table IV presents the relations that correspond to their composition $r_{1} \circ r_{2}(A, C, D)$. Similarly to Table II, we use $I M P$ to denote impossible cases.

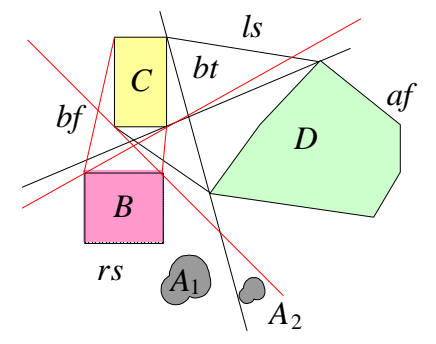

Fig. 9. Example of composition

Example 9: Assume that we want to compute the composition of $b f$ with $r s$. According to Table IV, we have:

$$
b f \circ r s=\delta(r s, a f)=\{r s, a f, r s: a f\} .
$$

To verify this equation, consider Figure 9. We have:

$$
\begin{array}{rrl}
r s \in b f \circ r s \quad \text { since } & b f\left(A_{1}, B, C\right), r s(B, C, D) \\
\text { and } & r s\left(A_{1}, C, D\right) . \\
\text { since } & b f\left(A_{2}, B, C\right), r s(B, C, D) \\
\text { and } & a f\left(A_{2}, C, D\right) . \\
r s: a f \in b f \circ r s \quad \text { since } & b f\left(A_{1} \cup A_{2}, B, C\right), r s(B, C, D) \\
\text { and } & r s: a f\left(A_{1} \cup A_{2}, C, D\right) .
\end{array}
$$

Also notice that for all relations $q \notin \delta(r s, a f)$ it is impossible to find configurations such that $q(A, C, D), b f(A, B, C)$ and $r s(B, C, D)$ simultaneously hold.
To compute the composition results of Table IV, we proceed as follows. For every pair of a single-tile relation $r_{1}$ and a basic relation $r_{2}$, we consider every possible basic relation $r_{3}$ and check if there exist regions $A, B, C$ and $D$ such that

$$
r_{1}(A, B, C), r_{2}(B, C, D) \text { and } r_{3}(A, C, D)
$$

hold. If we can find such regions then, according to Definition 3, $r_{3} \in r_{1} \circ r_{2}$ holds and thus $r_{3}$ is added in the $\left(r_{1}, r_{2}\right)$ entry of Table IV. Notice that, for each entry of Table IV, in the worst case we have to consider 34 (i.e., the total number of projective relations) configurations involving regions $A, B$, $C$ and $D$. To assist this procedure, we have implemented Algorithm COMPUTECOMPOSITION (Figure 10). Given four regions $R_{1}, R_{2}, R_{3}$ and $R_{4}$, the above algorithm computes relation $r_{1}\left(R_{1}, R_{2}, R_{3}\right)$ and $r_{2}\left(R_{2}, R_{3}, R_{4}\right)$ and their composition $r_{3}\left(R_{1}, R_{3}, R_{4}\right)$. Similarly to the rotation operation, regions $R_{1}, R_{2}, R_{3}$ and $R_{4}$ are constructed by the union of two rectangles (Step 3) that are taken from a large array of random rectangles $\mathcal{R}$ (Step 1). In our experiments, we have varied the size of array $\mathcal{R}$ from 100,000 to $1,000,000$.

\section{Algorithm COMPUTECOMPOSITION Method: \\ 1. Create an array $\mathcal{R}[1 \ldots n]$ that contains $n$ random rectangles \\ 2. For $i=1$ To $n-7$ \\ 3. Set $R_{1}=\mathcal{R}[i] \cup \mathcal{R}[i+1], R_{2}=\mathcal{R}[i+2] \cup \mathcal{R}[i+3]$, $R_{3}=\mathcal{R}[i+4] \cup \mathcal{R}[i+5], R_{4}=\mathcal{R}[i+6] \cup \mathcal{R}[i+7]$ \\ 4. Compute relations $r_{1}\left(R_{1}, R_{2}, R_{3}\right), r_{2}\left(R_{2}, R_{3}, R_{4}\right)$ and $r_{3}\left(R_{1}, R_{3}, R_{4}\right)$. \\ 5. ADD the $r_{3}$ to the composition entry of $r_{1} \circ r_{2}$ \\ 6. EndFor \\ // Since $r_{3} \in r_{1} \circ r_{2}$}

Fig. 10. Algorithm ComputeComposition

Similarly to the discussion about rotation, the results that Algorithm COMPUTECOMPOSITION produces are definitely sound since for every result there is an actual configuration of regions satisfying relations and their composition. As part of the proof, we manually drew all the configurations (reported in the Appendix). An empirical support to the completeness of Table IV is given by the fact that continuing to run the experiments no other results were found. The automatic procedure can also be used to find results for the full composition table of two basic relations (34 times 34 table), which is treated in Section VII-C.

\section{Composing basic relations}

Let us consider two basic relations $r$ and $q$ and let us assume that $r=r_{1}: \cdots: r_{k}$. In this section, we will reduce the computation of $r \circ q$ to the computation of compositions $r_{1} \circ q, \ldots$, $r_{k} \circ q$. All these expressions denote the composition of a singletile with a basic relation and can be computed using Table IV (see also Section VII-C). A natural method to perform this reduction is to use the product of relations (Definition 12). Specifically, we may use the expression $\pi\left(r_{1} \circ q, \ldots, r_{k} \circ q\right)$. We will refer to this expression as the product expression.

The product expression correctly computes the composition of relation in:ou as the following lemma demonstrates. 


\begin{tabular}{|c|c|c|c|c|c|c|c|}
\hline$r_{2} \backslash r_{1}$ & $b t$ & $r s$ & $b f$ & $l s$ & $a f$ & $i n$ & ou \\
\hline$\overline{\overline{b t}}$ & $\overline{\overline{b t}}$ & 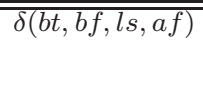 & $\begin{array}{l}\delta(b t, r s, l s, a f) \cup \\
\delta(b t, b f, l s, a f) \cup \\
\delta(b t, r s, b f, a f)\end{array}$ & 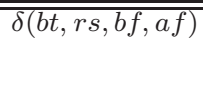 & $\begin{array}{l}\delta(b t, r s, b f, l s) \cup \\
\delta(b t, r s, b f, a f) \cup \\
\delta(b t, b f, l s, a f)\end{array}$ & $\overline{\overline{b t}}$ & $\overline{\overline{D_{d}}}$ \\
\hline$r s$ & $\delta(b t, r s, b f)$ & $\delta(b t, r s, l s, a f)$ & $\delta(r s, a f)$ & $\delta(r s, b f, l s)$ & $\delta(b t, b f, l s)$ & $\delta(b t, r s, b f)$ & $D_{d}$ \\
\hline$\overline{b f}$ & $\delta(b t, b f)$ & $\delta(b t, r s, b f, a f)$ & $\begin{array}{ll}\delta(r s, b f, l s) & \cup \\
\delta(r s, b f, a f) & \cup \\
\delta(b f, l s, a f) & \end{array}$ & $\delta(b t, b f, l s, a f)$ & $D_{d}$ & $\delta(b t, b f)$ & $\overline{D_{d}}$ \\
\hline$l s$ & $\delta(b t, b f, l s)$ & $\delta(r s, b f, l s)$ & $\delta(l s, a f)$ & $\delta(b t, r s, l s, a f)$ & $\delta(b t, r s, b f)$ & $\delta(b t, b f, l s)$ & $D_{d}$ \\
\hline$a f$ & $D_{d}$ & $\delta(b t, b f, l s, a f)$ & $\delta(b t, a f)$ & $\delta(b t, r s, b f, a f)$ & $\delta(b t, b f)$ & $I M P$ & $I M P$ \\
\hline$b t: r s$ & $\delta(b t, r s, b f, a f)$ & $\delta(b t, l s, a f)$ & $\begin{array}{l}\delta(b t, r s, l s, a f) \cup \\
\delta(b t, r s, b f, a f)\end{array}$ & $\delta(r s, b f)$ & $\begin{array}{l}\delta(b t, r s, b f, l s) \cup \\
\delta(b t, b f, l s, a f)\end{array}$ & $\delta(b t, r s, b f, a f)$ & $D_{d}$ \\
\hline$b t: b f$ & $\begin{array}{l}\delta(b t, r s, b f, a f) \cup \\
\delta(b t, b f, l s, a f)\end{array}$ & $\delta(b t, a f) \cup b f$ & $\begin{array}{l}\delta(b t, r s, b f, a f) \cup \\
\delta(b t, b f, l s, a f)\end{array}$ & $\delta(b t, a f) \cup b f$ & $\begin{array}{l}\delta(b t, b f, l s, a f) \cup \\
\delta(b t, r s, b f, a f)\end{array}$ & $D_{d}$ & $D_{d}$ \\
\hline$b t: l s$ & $\delta(b t, b f, l s, a f)$ & $\delta(b f, l s)$ & $\begin{array}{l}\delta(b t, r s, l s, a f) \cup \\
\delta(b t, b f, l s, a f)\end{array}$ & $\overline{\delta(b t, r s, a f)}$ & $\begin{array}{l}\delta(b t, r s, b f, l s) \cup \\
\delta(b t, r s, b f, a f)\end{array}$ & $\delta(b t, b f, l s, a f)$ & $D_{d}$ \\
\hline$\overline{b t: a f}$ & $D_{d}$ & $\delta(b t, b f, l s, a f)$ & $\begin{array}{l}\delta(b t, r s, l s, a f) \cup \\
\delta(b t, b f, l s, a f) \cup \\
\delta(b t, r s, b f, a f)\end{array}$ & $\delta(b t, r s, b f, a f)$ & $\begin{array}{l}\delta(b t, r s, b f, l s) \cup \\
\delta(b t, r s, b f, a f) \cup \\
\delta(b t, b f, l s, a f)\end{array}$ & $D_{d}$ & $D_{d}$ \\
\hline$r s: b f$ & $\delta(b t, r s, b f)$ & $\overline{\delta(b t, r s, a f)}$ & $\begin{array}{l}\delta(r s, b f, l s) \\
\delta(r s, b f, a f)\end{array}$ & $\delta(b f, l s)$ & $D_{d}$ & $\overline{\delta(b t, r s, b f)}$ & $D_{d}$ \\
\hline$r s: l s$ & $D_{d}$ & $\{r s, l s\}$ & $\begin{array}{l}\delta(b t, r s, l s, a f) \cup \\
\delta(r s, b f, l s)\end{array}$ & $\{r s, l s\}$ & $\begin{array}{l}\delta(b t, r s, l s, a f) \cup \\
\delta(r s, b f, l s)\end{array}$ & $D_{d}$ & $D_{d}$ \\
\hline$r s: a f$ & $D_{d}$ & $\delta(b t, l s, a f)$ & $\delta(b t, r s, a f)$ & $\delta(r s, b f)$ & $\delta(b t, b f, l s)$ & $D_{d}$ & $D_{d}$ \\
\hline$b f: l s$ & $\delta(b t, b f, l s)$ & $\delta(r s, b f)$ & $\begin{array}{l}\delta(r s, b f, l s) \\
\delta(b f, l s, a f)\end{array}$ & $\delta(b t, l s, a f)$ & $D_{d}$ & $\delta(b t, b f, l s)$ & $D_{d}$ \\
\hline$b f: a f$ & $D_{d}$ & $\delta(b t, a f) \cup b f$ & $\begin{array}{l}\delta(b t, r s, b f, a f) \cup \\
\delta(b t, b f, l s, a f)\end{array}$ & $\delta(b t, a f) \cup b f$ & $\begin{array}{l}\delta(b t, r s, b f, a f) \cup \\
\delta(b t, b f, l s, a f)\end{array}$ & $D_{d}$ & $D_{d}$ \\
\hline ls:af & $D_{d}$ & $\delta(b f, l s)$ & $\delta(b t, l s, a f)$ & $\delta(b t, r s, a f)$ & $\delta(b t, r s, b f)$ & $D_{d}$ & $D_{d}$ \\
\hline$b t: r s: b f$ & $\delta(b t, r s, b f, a f)$ & $\delta(b t, a f)$ & $\delta(b t, r s, b f, a f)$ & $b f$ & $\delta(b t, b f, l s, a f)$ & $D_{d}$ & $D_{d}$ \\
\hline bt:rs:ls & $D_{d}$ & $l s$ & $\delta(b t, r s, l s, a f)$ & $r s$ & $\delta(r s, b f, l s)$ & $D_{d}$ & $D_{d}$ \\
\hline$b t: r s: a f$ & $D_{d}$ & $\delta(b t, l s, a f)$ & $\begin{array}{l}\delta(b t, r s, l s, a f) \cup \\
\delta(b t, r s, b f, a f)\end{array}$ & $\delta(r s, b f)$ & $\begin{array}{l}\delta(b t, r s, b f, l s) \cup \\
\delta(b t, b f, l s, a f)\end{array}$ & $D_{d}$ & $D_{d}$ \\
\hline$b t: b f: l s$ & $\delta(b t, b f, l s, a f)$ & $b f$ & $\delta(b t, b f, l s, a f)$ & $\delta(b t, a f)$ & $\delta(b t, r s, b f, a f)$ & $D_{d}$ & $D_{d}$ \\
\hline$b t: b f: a f$ & $D_{d}$ & $\delta(b t, a f) \cup \mathrm{bf}$ & $\begin{array}{l}\delta(b t, r s, b f, a f) \cup \\
\delta(b t, b f, l s, a f)\end{array}$ & $\delta(b t, a f) \cup b f$ & $\begin{array}{l}\delta(b t, r s, b f, a f) \cup \\
\delta(b t, b f, l s, a f)\end{array}$ & $D_{d}$ & $D_{d}$ \\
\hline$b t: l s: a f$ & $D_{d}$ & $\delta(b f, l s)$ & $\begin{array}{l}\delta(b t, r s, l s, a f) \cup \\
\delta(b t, b f, l s, a f)\end{array}$ & $\overline{\delta(b t, r s, a f)}$ & $\begin{array}{l}\delta(b t, r s, b f, l s) \cup \\
\delta(b t, r s, b f, a f)\end{array}$ & $D_{d}$ & $D_{d}$ \\
\hline$r s: b f: l s$ & $\delta(b t, r s, b f, l s)$ & rs & $\delta(r s, b f, l s)$ & $l s$ & $\delta(b t, r s, l s, a f)$ & $D_{d}$ & $D_{d}$ \\
\hline$r s: b f: a f$ & $D_{d}$ & $\delta(b t, a f)$ & $\delta(b t, r s, b f, a f)$ & $b f$ & $\delta(b t, b f, l s, a f)$ & $D_{d}$ & $D_{d}$ \\
\hline$r s: l s: a f$ & $D_{d}$ & $l s$ & $\delta(b t, r s, l s, a f)$ & $r s$ & $\delta(r s, b f, l s)$ & $D_{d}$ & $D_{d}$ \\
\hline$b f: l s: a f$ & $D_{d}$ & $b f$ & $\delta(b t, b f, l s, a f)$ & $\delta(b t, a f)$ & $\delta(b t, r s, b f, a f)$ & $D_{d}$ & $D_{d}$ \\
\hline$b t: r s: b f: l s$ & $I M P$ & $I M P$ & $I M P$ & $I M P$ & $I M P$ & $D_{d}$ & $D_{d}$ \\
\hline$b t: r s: b f: a f$ & $D_{d}$ & $\delta(b t, a f)$ & $\delta(b t, r s, b f, a f)$ & $b f$ & $\delta(b t, b f, l s, a f)$ & $D_{d}$ & $D_{d}$ \\
\hline$b t: r s: l s: a f$ & $D_{d}$ & $l s$ & $\delta(b t, r s, l s, a f)$ & $r s$ & $\delta(r s, b f, l s)$ & $D_{d}$ & $D_{d}$ \\
\hline$b t: b f: l s: a f$ & $D_{d}$ & $b f$ & $\delta(b t, b f, l s, a f)$ & $\delta(b t, a f)$ & $\delta(b t, r s, b f, a f)$ & $D_{d}$ & $D_{d}$ \\
\hline$r s: b f: l s: a f$ & $I M P$ & $I M P$ & $I M P$ & $I M P$ & $I M P$ & $D_{d}$ & $D_{d}$ \\
\hline$b t: r s: b f: l s: a f$ & $I M P$ & $I M P$ & $I M P$ & $I M P$ & $I M P$ & $\overline{D_{d}}$ & $\overline{D_{d}}$ \\
\hline in & in & $D_{c}$ & $D_{c}$ & $D_{c}$ & $D_{c}$ & in & $D_{c}$ \\
\hline ou & $D_{c}$ & $D_{c}$ & ou & $D_{c}$ & $D_{c}$ & $D_{c}$ & $D_{c}$ \\
\hline in:ou & $D_{c}$ & $D_{c}$ & $D_{c}$ & $D_{c}$ & $D_{c}$ & $D_{c}$ & $D_{c}$ \\
\hline
\end{tabular}

TABLE IV

COMPOSING A SINGLE-TILE WITH A BASIC RELATION

Lemma 1: Let $q$ is a basic relation. The composition of relation in:ou and $q$ can be computed using the following formula:

$$
\text { in:ou } \circ q=\pi(\text { in } \circ q, \text { ou } \circ q) .
$$

For example, using Lemma 1, we have

$$
\begin{aligned}
\text { in:ou } \circ \mathrm{rs} & =\pi(\text { in } \circ r s, \text { ou } \circ r s) \\
& =\pi\left(\delta(b t, r s, b f), D_{d}\right) \\
& =D_{d} .
\end{aligned}
$$

This result can be verified using Figure 11. Notice that $r s(B, C, D)$ holds. To compute the composition we should investigate the possible position of a region $A$ such that in:ou $(A, B, C)$ holds. This means that $A$ has a part that is inside and a part that is outside $C H(B \cup C)$. It is not hard to verify that in general $D_{d}(A, C, D)$ holds. For instance, if $A=A_{1} \cup A_{2}$ then bt: $\operatorname{f} f(A, C, D)$ holds. 


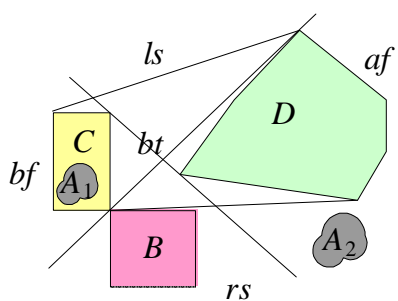

Fig. 11. Composing in:ou and $r s$

Let us now investigate the relation between composition $(r \circ q)$ and the product expression $\left(\pi\left(r_{1} \circ q, \ldots, r_{k} \circ q\right)\right)$ in the case of the remaining ternary projective relations. Consider the following example.

Example 10: Let us consider relations bt:rs and bt:bf. According to Table IV, we have:

$$
\begin{aligned}
b t \circ b t: b f & =\delta(b t, r s, b f, a f) \cup \delta(b t, b f, l s, a f) \text { and } \\
r s \circ b t: b f & =\delta(b t, a f) \cup b f .
\end{aligned}
$$

Thus, the product expression gives:

$$
\begin{aligned}
& \pi(b t \circ b t: b f, r s \circ b t: b f)= \\
& \pi(\delta(b t, r s, b f, a f) \cup \delta(b t, b f, l s, a f), \delta(b t, a f) \cup b f)= \\
& \pi\left(\begin{array}{c}
b t, r s, b f, l s, a f, \\
b t: r s, b t: b f, b t: l s, \\
b t: a f, r s: b f, r s: a f, \\
b f: l s, b f: a f, l s: a f, \\
b t: r s: b f, b t: r s: a f, \\
b t: b f: l s, b t: b f: a f, \\
b t: l s: a f, r s: b f: a f, \\
b f: l s: a f, b t: r s: b f: a f, \\
b t: b f: l s: a f \\
\quad b f, a f, b t: r s, b t: b f, b t: l s, b t: a f, r s: b f, \\
r s: a f, b f: l s, b f: a f, l s: a f, b t: r s: b f, \\
b t: r s: a f, b t: b f: l s, b t: b f: a f, b t: l s: a f, \\
r s: b f: a f, b f: l s: a f, b t: r s: b f: a f, b t: b f: l s: a f
\end{array}\right\} .
\end{aligned}
$$

The above expression does not correctly compute the composition. For instance, $b t: l s \in \pi(b t \circ b t: b f, r s \circ b t: b f)$ but $b t: l s \notin b t: r s \circ b t: b f$ because there cannot be regions $A$, $B, C$ and $D$ such that $b t: l s(A, C, D)$, bt:rs $(A, B, C)$ and $b t: b f(B, C, D)$ hold at the same time.

In Example 10, we have seen that the product expression contains relations that do not belong to the composition. We can prove that a possible result of the composition operator is necessarily included in the product expression but the product expression may contain relations that do not belong to the composition. This fact is captured in the following proposition.

Proposition 3: Let $r_{1}: \cdots: r_{k}$ and $q$ be two projective relations. Then, we have:

$$
\left(r_{1}: \cdots: r_{k}\right) \circ q \subseteq \pi\left(r_{1} \circ q, \ldots, r_{k} \circ q\right) .
$$

Proof: We will first prove that if $u \in r_{1}: \cdots: r_{k} \circ q$ then $u \in \pi\left(r_{1} \circ q, \ldots, r_{k} \circ q\right)$.

Since $u \in r_{1}: \cdots: r_{k} \circ q$ holds, there are regions $A, B, C$ and $D$ such that

$$
r_{1}: \cdots: r_{k}(A, B, C) \wedge q(B, C, D) \wedge u(A, C, D) .
$$

Since, $r_{1}: \cdots: r_{k}(A, B, C)$ holds, according to Definition 6, there are regions $A_{1}, \ldots, A_{k}$ such that $A=A_{1} \cup \cdots \cup A_{k}$ and $r_{1}\left(A_{1}, B, C\right), \ldots, r_{k}\left(A_{k}, B, C\right)$ hold. Thus, we have:

$r_{1}\left(A_{1}, B, C\right) \wedge \cdots \wedge r_{k}\left(A_{k}, B, C\right) \wedge q(B, C, D) \wedge u(A, C, D)$.

Now, let $u_{1}$ be the relation that holds between regions $A_{1}, C$ and $D$, i.e., $u_{1}\left(A_{1}, C, D\right)$. Similarly, we define $u_{2}\left(A_{2}, C, D\right)$, $\ldots, u_{k}\left(A_{k}, C, D\right)$. Notice that $u=\operatorname{tile-union}\left(u_{1}, \ldots, u_{k}\right)$ holds, thus we have:

$$
\begin{gathered}
r_{1}\left(A_{1}, B, C\right) \wedge \cdots \wedge r_{k}\left(A_{k}, B, C\right) \wedge \\
q(B, C, D) \wedge u_{1}\left(A_{1}, C, D\right) \wedge \cdots \wedge u_{k}\left(A_{k}, C, D\right)
\end{gathered}
$$

and by rewriting the expression we have:

$$
\begin{gathered}
r_{1}\left(A_{1}, B, C\right) \wedge q(B, C, D) \wedge u_{1}\left(A_{1}, C, D\right) \\
\wedge \cdots \wedge \\
r_{k}\left(A_{k}, B, C\right) \wedge q(B, C, D) \wedge u_{k}\left(A_{k}, C, D\right) .
\end{gathered}
$$

Summarizing, according to Definition 3, we have $u_{1} \in r_{1} \circ q$, $\ldots, u_{k} \in r_{k} \circ q$ and $u=\operatorname{tile-union}\left(u_{1}, \ldots, u_{k}\right)$, thus, $u \in$ $\pi\left(r_{1} \circ q, \ldots, r_{k} \circ q\right)$ (see also Definition 12). To conclude this proof, we notice that Example 10 illustrates a case where $u \in \pi\left(r_{1} \circ q, \ldots, r_{k} \circ q\right)$ and $u \notin r \circ q$.

In total the product expression, although it seems to be a natural choice, results in a superset of the composition result. It is interesting to identify the cases where the product expression produces a result that does not belong to the composition. To this end, let us reconsider Example 10. The composition of $b t: r s$ with $b t: b f$ is given by the following formula.

$$
b t: r s \circ b t: b f=\left\{\begin{array}{c}
b t, b f, a f, b t: r s, b t: b f, b t: a f, \\
r s: a f, b f: l s, b f: a f, b t: r s: b f, \\
b t: r s: a f, b t: b f: l s, b t: b f: a f, \\
r s: b f: a f, b f: l s: a f, \\
b t: r s: b f: a f, b t: b f: l s: a f
\end{array}\right\}
$$

To verify this expression we consider Figure 12. In this figure, we present two configurations involving three regions $B, C$ and $D$ such that $b t: b f(B, C, D)$. In both configurations of Figure 12, a region $A$ satisfies relation $b t: r s(A, B, C)$ iff it has a part that lies in the dark-shaded area and a part that lies in the light-shaded area. It is not hard to verify that all the possible relations that hold between $A, C$ and $D$ are prescribed by the previous expression. Also notice that for all relations $q$ that are not mentioned in the set of Equation 1 it is impossible to find configurations such that $q(A, C, D), b t: r s(A, B, C)$ and $b t: b f(B, C, D)$ simultaneously hold.

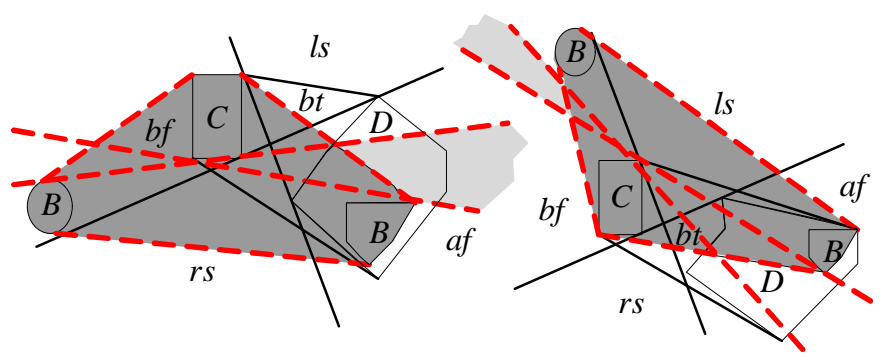

(a)

(b)

Fig. 12. Composing bt:rs with $b t: b f$ 
Equation 1 can be equivalently written as follows.

$$
\begin{gathered}
b t: r s \circ b t: b f=\pi(\delta(b t, r s, b f, a f), \delta(b t, a f)) \cup \\
\pi(\delta(b t, b f, l s, a f), b f)
\end{gathered}
$$

Let us compare the above expression with the product expression of Example 10, i.e.,

$$
\begin{gathered}
\pi(b t \circ b t: b f, r s \circ b t: b f)= \\
\pi(\delta(b t, r s, b f, a f) \cup \delta(b t, b f, l s, a f), \delta(b t, a f) \cup b f) .
\end{gathered}
$$

Using Proposition 1, we have:

$$
\begin{gathered}
\pi(b t \circ b t: b f, r s \circ b t: b f)=\pi(\delta(b t, r s, b f, a f), \delta(b t, a f)) \cup \\
\pi(\delta(b t, b f, l s, a f), b f) \cup \pi(\delta(b t, r s, b f, a f), b f) \cup \\
\pi(\delta(b t, b f, l s, a f), \delta(b t, a f))
\end{gathered}
$$

and

$$
\begin{gathered}
\pi(b t \circ b t: b f, r s \circ b t: b f)=b t: r s \circ b t: b f \cup \\
\pi(\delta(b t, r s, b f, a f), b f) \cup \pi(\delta(b t, b f, l s, a f), \delta(b t, a f)) .
\end{gathered}
$$

In other words, to get the correct composition result we should rule out some combinations that appear in the product expression (see also Example 10 and Proposition 3). These combinations correspond to impossible configurations. In our example, it is not possible to find regions $A$, $B, C$ and $D$ such that $r(A, C, D)$, bt:rs $(A, B, C)$ and $b t: b f(B, C, D)$ for any relation $r \in \pi(\delta(b t, r s, b f, a f), b f) \cup$ $\pi(\delta(b t, b f, l s, a f), \delta(b t, a f)$. This can be verified using Figure 12 .

Inspired by this observation, we refine Table IV that presents the composition of a single-tile with a basic relation. First, for each entry of the table, we consider the possible configurations that produce the composition result. Then, for each row of the table we group the compatible configurations in the same row. The new results appear in Tables V and VI. In total, these tables present the result of the composition of a singletile relation $r_{1}$ with a basic (single or multi-tile) relation $r_{2}$. These tables have 34 rows (numbered from 1 to 34 ) that correspond to the basic relation $r_{2}$. Each row is divided into several subrows. For instance, the rows numbered 1 and 13 are divided into 4 and 3 subrows respectively. The composition of relations $r_{1}$ and $r_{2}$ is computed by the union of the subrows that lie in on the crossing of the row title $r_{1}$ with the column title $r_{2}$. It is easy to verify that Table IV can be produced by Tables V and VI by unifying the subrows of every cell. For instance, using Table $\mathrm{V}$ we have:

$$
\begin{aligned}
b f \circ b t= & \delta(b t, a f) \cup \delta(b t, r s, l s, a f) \cup \delta(b t, b f, l s, a f) \cup \\
& \delta(b t, r s, b f, a f) \\
= & \delta(b t, r s, l s, a f) \cup \delta(b t, b f, l s, a f) \cup \\
& \delta(b t, r s, b f, a f)
\end{aligned}
$$

This is exactly the result we get by using Table IV.

Each subrow of Tables $\mathrm{V}$ and VI corresponds to a possible configuration. Results taken in different subrows of the same row correspond to impossible configurations. For instance, to compute the composition of bt:rs and bt:bf we consider the elements that lie on the crossing of the 7th row of Table $\mathrm{V}$ with columns title $b t$ and $r s$. To get the result, we take the union of the product of the elements of all subrows. In total, we have:

$$
\begin{gathered}
b t: r s \circ b t: b f=\pi(\delta(b t, r s, b f, a f), \delta(b t, a f)) \cup \\
\pi(\delta(b t, b f, l s, a f), b f)
\end{gathered}
$$

which is exactly the result of Equation 2.

As another example, the composition of bt:ls:af with $b t: r s: a f$ is given by the following formula.

$$
\begin{aligned}
& b t: l s: a f \circ b t: r s: a f= \\
& \pi\left(D_{d}, \delta(r s, b f), \delta(b f, l s)\right) \cup \\
& \pi(\delta(b t, r s, b f, a f), \delta(r s, b f), \delta(b t, b f, l s)) \cup \\
& \pi(\delta(b t, r s, b f, a f), r s, \delta(b t, r s, b f, l s)) \cup \\
& \pi(\delta(b t, r s, b f, a f), b f, \delta(b t, b f, l s, a f)) \cup \\
& \pi\left(D_{d}, r s, \delta(r s, b f, l s)\right)
\end{aligned}
$$

To compute Tables V and VI we consider one row at a time. For each row, we consider all different configurations that are able to reproduce all the composition results that appear for the respective row in Table IV. Each configuration corresponds to a subrow. For example, let us consider the first row of Table V, i.e., relation $b t$. To complete this row, we consider different configurations of regions $B, C$ and $D$ such that $b t(B, C, D)$ holds. To reproduce the results of Table IV, we need four different configurations that correspond to the four different subrows of Table V. These configurations appear in Figure 13. For instance, in Figure 13a:

1) For all regions $A$ such that $b t(A, B, C)$, we have $b t(A, C, D)$.

2) For all regions $A$ such that $\operatorname{rs}(A, B, C)$, we have $\delta(b t, b f, l s, a f)(A, C, D)$.

3) For all regions $A$ such that $b f(A, B, C)$, we have $\delta(b t, a f)(A, C, D)$.

4) For all regions $A$ such that $l s(A, B, C)$, we have $\delta(b t, r s, b f, a f)(A, C, D)$.

5) For all regions $A$ such that $a f(A, B, C)$, we have $\delta(b t, b f)(A, C, D)$.

All these results are captured in the $(a)$ subrow of $b t$. Similarly, using Figures $13 \mathrm{~b}-\mathrm{d}$, we can verify all the other subrows of bt.

The configurations that we consider for each row are a minimal covering set: this assures that no results in the composition are missing and that impossible results are filtered out. The configurations that verify all the other rows of Tables V and VI are presented in the Appendix.

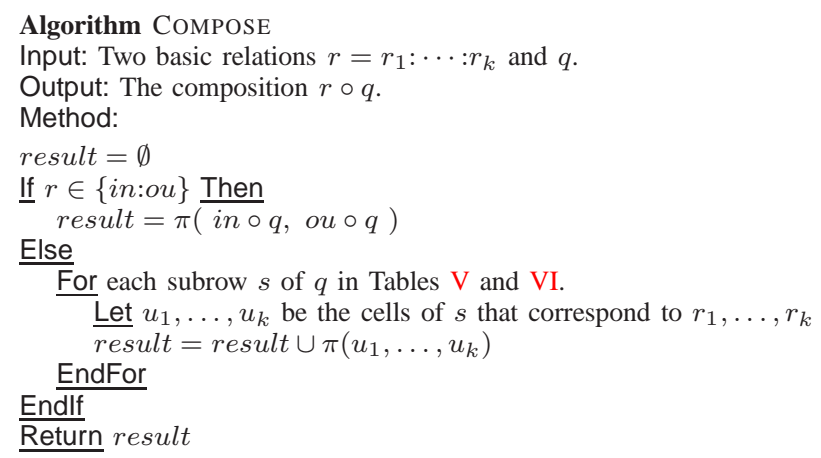

Fig. 14. Algorithm Compose

Overall, we obtain a procedure to find the composition of two basic relations starting from the knowledge of the composition of a single-tile relation with a basic relation. Such a procedure is summarized in Algorithm COMPOSE presented 


\begin{tabular}{|c|c|c|c|c|c|c|c|}
\hline & $r_{2} \backslash r_{1}$ & & $b t$ & $r s$ & $b f$ & $l s$ & $a f$ \\
\hline \multirow[t]{4}{*}{1} & \multirow[t]{4}{*}{$\overline{b t}$} & $\overline{\bar{a} a}$ & $\overline{\overline{b t}}$ & $\overline{c \delta(b t, b f, l s, a f)}$ & $\overline{\bar{\delta}(b t, a f)}$ & $\bar{c} \delta(b t, r s, b f, a f)$ & $\overline{\delta \delta(b t, b f)}$ \\
\hline & & $b$ & $b t$ & $\delta(b t, l s)$ & $\delta(b t, r s, l s, a f)$ & $\delta(b t, r s)$ & $\delta(b t, r s, b f, l s)$ \\
\hline & & $c$ & $b t$ & $\delta(b t, b f)$ & $\delta(b t, b f, l s, a f)$ & $\delta(b t, a f)$ & $\delta(b t, r s, b f, a f)$ \\
\hline & & $d$ & $b t$ & $\delta(b t, a f)$ & $\delta(b t, r s, b f, a f)$ & $\delta(b t, b f)$ & $\delta(b t, b f, l s, a f)$ \\
\hline \multirow[t]{2}{*}{2} & \multirow[t]{2}{*}{$r s$} & $a$ & $\overline{c \delta(b t, r s, b f)}$ & $\overline{l \delta(b t, r s, l s, a f)}$ & $\overline{\delta(r s, a f)}$ & $\overline{\delta(r s, b f)}$ & $\bar{\delta} \delta(b t, b f, l s)$ \\
\hline & & $b$ & $\delta(b t, r s, b f)$ & $\delta(b t, r s, l s, a f)$ & $r s$ & $\delta(r s, b f, l s)$ & $\delta(b t, b f, l s)$ \\
\hline \multirow[t]{4}{*}{3} & \multirow[t]{4}{*}{$b f$} & $\bar{a}$ & $\delta(b t, b f)$ & $\delta(r s, b f)$ & $\delta(r s, b f, l s)$ & $\delta(b f, l s)$ & $D_{d}$ \\
\hline & & $b$ & $\delta(b t, b f)$ & $\delta(b t, r s, b f, a f)$ & $\delta(r s, b f, a f)$ & $b f$ & $\delta(b t, b f, l s, a f)$ \\
\hline & & $c$ & $\delta(b t, b f)$ & $b f$ & $\delta(b f, l s, a f)$ & $\delta(b t, b f, l s, a f)$ & $\delta(b t, r s, b f, a f)$ \\
\hline & & $d$ & $\delta(b t, b f)$ & $\delta(b t, r s, b f, a f)$ & $b f$ & $\delta(b t, b f, l s, a f)$ & $\delta(b t, a f)$ \\
\hline \multirow[t]{2}{*}{4} & \multirow[t]{2}{*}{$l s$} & $a$ & $\delta(b t, b f, l s)$ & $\delta(b f, l s)$ & $\delta(l s, a f)$ & $\delta(b t, r s, l s, a f)$ & $\delta(b t, r s, b f)$ \\
\hline & & $b$ & $\delta(b t, b f, l s)$ & $\delta(r s, b f, l s)$ & Ts & $\delta(b t, r s, l s, a f)$ & $\delta(b t, r s, b f)$ \\
\hline \multirow[t]{4}{*}{5} & \multirow[t]{4}{*}{$\overline{a f}$} & $a$ & $\overline{D_{d}}$ & $\overline{c \delta(b f, l s, a f)}$ & $\overline{\delta(b t, a f)}$ & $\overline{\delta(r s, b f, a f)}$ & $\overline{b f}$ \\
\hline & & $b$ & $\delta(b t, a f)$ & $\delta(b t, b f, l s, a f)$ & $\delta(b t, a f)$ & $\delta(b t, r s, b f, a f)$ & $\begin{array}{l}\delta(b t, b f) \\
\end{array}$ \\
\hline & & $c$ & $\delta(b t, r s, b f, a f)$ & $\delta(b t, b f, l s, a f)$ & $\delta(b t, a f)$ & $\delta(r s, b f, a f)$ & $\delta(b t, b f)$ \\
\hline & & $d$ & $\delta(b t, b f, l s, a f)$ & $\delta(b f, l s, a f)$ & $\delta(b t, a f)$ & $\delta(b t, r s, b f, a f)$ & $\begin{array}{l}\delta(b t, b f) \\
\end{array}$ \\
\hline \multirow[t]{3}{*}{6} & \multirow[t]{3}{*}{$b t: r s$} & $a$ & $\delta(b t, r s, b f, a f)$ & $\delta(b t, l s)$ & $\delta(b t, r s, l s, a f)$ & $r s$ & $\delta(b t, r s, b f, l s)$ \\
\hline & & $b$ & $\delta(b t, r s, b f, a f)$ & $\delta(b t, l s, a f)$ & $\delta(b t, r s, a f)$ & $\delta(r s, b f)$ & $\delta(b t, b f, l s)$ \\
\hline & & $c$ & $\delta(b t, r s, b f, a f)$ & $\delta(b t, a f)$ & $\delta(b t, r s, b f, a f)$ & $b f$ & $\delta(b t, b f, l s, a f)$ \\
\hline \multirow[t]{2}{*}{7} & \multirow[t]{2}{*}{$b t: b f$} & $a$ & $\delta(b t, r s, b f, a f)$ & $\delta(b t, a f)$ & $\delta(b t, r s, b f, a f)$ & $b f$ & $\delta(b t, b f, l s, a f)$ \\
\hline & & $b$ & $\delta(b t, b f, l s, a f)$ & $b f$ & $\delta(b t, b f, l s, a f)$ & $\delta(b t, a f)$ & $\delta(b t, r s, b f, a f)$ \\
\hline \multirow[t]{3}{*}{8} & \multirow[t]{3}{*}{ bt:ls } & $a$ & $\delta(b t, b f, l s, a f)$ & $l s$ & $\delta(b t, r s, l s, a f)$ & $\delta(b t, r s)$ & $\delta(b t, r s, b f, l s)$ \\
\hline & & $b$ & $\delta(b t, b f, l s, a f)$ & $\delta(b f, l s)$ & $\delta(b t, l s, a f)$ & $\delta(b t, r s, a f)$ & $\delta(b t, r s, b f)$ \\
\hline & & $c$ & $\delta(b t, b f, l s, a f)$ & $b f$ & $\delta(b t, b f, l s, a f)$ & $\delta(b t, a f)$ & $\delta(b t, r s, b f, a f)$ \\
\hline \multirow[t]{7}{*}{$\overline{\overline{9}}$} & \multirow[t]{7}{*}{$\overline{c b t: a f}$} & $\bar{a}$ & $\overline{\overline{D_{d}}}$ & $\overline{\bar{\delta}(b f, l s, a f)}$ & $\overline{\delta(b t, a f)}$ & $\overline{\delta(r s, b f, a f)}$ & $\overline{b f}$ \\
\hline & & $b$ & $\delta(b t, a f)$ & $\delta(b t, b f, l s, a f)$ & $\delta(b t, a f)$ & $\delta(b t, r s, b f, a f)$ & $\delta(b t, b f)$ \\
\hline & & $c$ & $\delta(b t, a f)$ & $\delta(b t, l s)$ & $\delta(b t, r s, l s, a f)$ & $\delta(b t, r s)$ & $\delta(b t, r s, b f, l s)$ \\
\hline & & $d$ & $\delta(b t, a f)$ & $\delta(b t, b f)$ & $\delta(b t, b f, l s, a f)$ & $\delta(b t, a f)$ & $\delta(b t, r s, b f, a f)$ \\
\hline & & $e$ & $\delta(b t, a f)$ & $\delta(b t, a f)$ & $\delta(b t, r s, b f, a f)$ & $\delta(b t, b f)$ & $\delta(b t, b f, l s, a f)$ \\
\hline & & $f$ & $\delta(b t, r s, b f, a f)$ & $\delta(b t, l s)$ & $\delta(b t, r s, l s, a f)$ & $r s$ & $\delta(b t, r s, b f, l s)$ \\
\hline & & $g$ & $\delta(b t, b f, l s, a f)$ & ts & $\delta(b t, r s, l s, a f)$ & $\delta(b t, r s)$ & $\delta(b t, r s, b f, l s)$ \\
\hline \multirow[t]{3}{*}{10} & \multirow[t]{3}{*}{$r s: b f$} & $\bar{a}$ & $\overline{\delta(b t, r s, b f)}$ & $\overline{\delta(b t, r s, a f)}$ & $\overline{\delta(r s, b f, a f)}$ & $\overline{b f}$ & $\delta(b t, b f, l s, a f)$ \\
\hline & & $b$ & $\delta(b t, r s, b f)$ & $r s$ & $\delta(r s, b f, l s)$ & $\delta(b f, l s)$ & $D_{d}$ \\
\hline & & $c$ & $\delta(b t, r s, b f)$ & $\delta(b t, r s, a f)$ & $\delta(r s, b f)$ & $\delta(b f, l s)$ & $\delta(b t, b f, l s, a f)$ \\
\hline \multirow[t]{2}{*}{11} & rrs:ls & $\bar{a} a$ & $\overline{D_{d}}$ & $\overline{l l s}$ & $\overline{l \delta(b t, r s, l s, a f)}$ & rss & $\overline{\delta \delta(r s, b f, l s)}$ \\
\hline & & $b$ & $\delta(b t, r s, b f, l s)$ & $r s$ & $\delta(r s, b f, l s)$ & $l s$ & $\delta(b t, r s, l s, a f)$ \\
\hline 12 & $r s: a f$ & $a$ & $D_{d}$ & $\delta(l s, a f)$ & $\delta(b t, r s, a f)$ & $\delta(r s, b f)$ & $\delta(b f, l s)$ \\
\hline & & $b$ & $\delta(b t, r s, b f, a f)$ & $\delta(b t, l s, a f)$ & $\delta(b t, r s, a f)$ & $\delta(r s, b f)$ & $\delta(b t, b f, l s)$ \\
\hline 13 & $b f: l s$ & $a$ & $\overline{\delta(b t, b f, l s)}$ & $\overline{b f}$ & $\overline{\delta(b f, l s, a f)}$ & $\overline{c \delta(b t, l s, a f)}$ & $\delta(b t, r s, b f, a f)$ \\
\hline & & $b$ & $\delta(b t, b f, l s)$ & $\delta(r s, b f)$ & $\delta(r s, b f, l s)$ & $l s$ & $D_{d}$ \\
\hline & & $c$ & $\delta(b t, b f, l s)$ & $\delta(r s, b f)$ & $\delta(b f, l s)$ & $\delta(b t, l s, a f)$ & $\delta(b t, r s, b f, a f)$ \\
\hline 14 & $b f: a f$ & $a$ & $\delta(b t, r s, b f, a f)$ & $\overline{\delta(b t, a f)}$ & $\bar{\delta}(b t, r s, b f, a f)$ & $\overline{b f}$ & $\delta(b t, b f, l s, a f)$ \\
\hline & & $b$ & $D_{d}$ & af & $\delta(b t, r s, b f, a f)$ & $b f$ & $\delta(b f, l s, a f)$ \\
\hline & & $c$ & $\delta(b t, b f, l s, a f)$ & $b f$ & $\delta(b t, b f, l s, a f)$ & $\delta(b t, a f)$ & $\delta(b t, r s, b f, a f)$ \\
\hline & & $d$ & $D_{d}$ & $b f$ & $\delta(b t, b f, l s, a f)$ & $a f$ & $\delta(r s, b f, a f)$ \\
\hline$\overline{15}$ & ls:af & $\bar{a} a$ & $\overline{D_{d}}$ & $\overline{\delta(b f, l s)}$ & $\overline{c \delta(b t, l s, a f)}$ & $\overline{\delta(r s, a f)}$ & $\overline{\delta(r s, b f)}$ \\
\hline & & $b$ & $\delta(b t, b f, l s, a f)$ & $\delta(b f, l s)$ & $\delta(b t, l s, a f)$ & $\delta(b t, r s, a f)$ & $\delta(b t, r s, b f)$ \\
\hline
\end{tabular}

TABLE V

COMPOSING A SINGLE-TILE WITH A BASIC RELATION (1/2)

in Figure 14. Furthermore, we tested that the results obtained by Algorithm COMPOSE coincide with the results obtained by Algorithm COMPUTEROTATION.

Theorem 1: Algorithm COMPOSE correctly computes the composition of two basic relations.

Proof: The proof follows from Lemma 1 and the previous discussion.

\section{CONCLUSIONS}

In this paper, we developed a reasoning system for ternary projective relations among regions of the plane. The reasoning system is based on three kinds of rules: converse, rotation, and composition. Converse and rotation are given in the form of tables for the 34 basic projective relations of our model. While the converse table is more immediate to find, the rotation table has been experimentally verified with test data. The composition table is the more involved to construct since it contains $34 \times 34$ entries. Instead of finding out each entry of the table individually, we tried to discover algebraic rules that were able to compute, at least partially, the results. Though it was impossible to find algebraic rules for the whole table, we could find that, starting from a subset of the table related to the composition of the 7 single-tile relations with all 34 relations, we can compute the remaining part of the table with a socalled product operation. Therefore, we found the $7 \times 34$ table both manually and experimentally by running the computation 


\begin{tabular}{|c|c|c|c|c|c|c|c|}
\hline & $r_{2} \backslash r_{1}$ & & $b t$ & $r s$ & $b f$ & $l s$ & $\overline{a f}$ \\
\hline 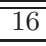 & $\overline{b t: r s: b f}$ & 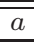 & $\overline{c \delta(b t, r s, b f, a f)}$ & $\delta(b t, a f)$ & 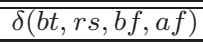 & $\overline{\overline{b f}}$ & 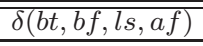 \\
\hline 17 & bt:rs:ls & $\bar{a}$ & $\overline{D_{d}}$ & $\overline{l s}$ & 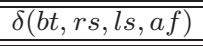 & rss & $\bar{c} \delta(r s, b f, l s)$ \\
\hline \multirow[t]{5}{*}{18} & \multirow[t]{5}{*}{$\overline{p b t: r s: a f}$} & $\overline{\bar{a} a}$ & 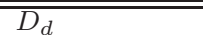 & $\overline{\bar{\rho} \delta(l s, a f)}$ & 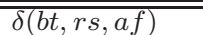 & $\overline{\bar{\delta}(r s, b f)}$ & $\overline{\bar{\delta}(b f, l s)}$ \\
\hline & & $b$ & $\delta(b t, r s, b f, a f)$ & $\delta(b t, l s, a f)$ & $\delta(b t, r s, a f)$ & $\delta(r s, b f)$ & $\delta(b t, b f, l s)$ \\
\hline & & $c$ & $\delta(b t, r s, b f, a f)$ & $\delta(b t, l s)$ & $\delta(b t, r s, l s, a f)$ & $r s$ & $\delta(b t, r s, b f, l s)$ \\
\hline & & $d$ & $\delta(b t, r s, b f, a f)$ & $\delta(b t, a f)$ & $\delta(b t, r s, b f, a f)$ & $b f$ & $\delta(b t, b f, l s, a f)$ \\
\hline & & $e$ & $D_{d}$ & ls & $\delta(b t, r s, l s, a f)$ & rs & $\delta(r s, b f, l s)$ \\
\hline$\overline{19}$ & $b t: b f: l s$ & $\bar{a}$ & $\delta(b t, b f, l s, a f)$ & $\overline{b f}$ & $\bar{\delta}(b t, b f, l s, a f)$ & $\overline{\delta(b t, a f)}$ & $\bar{\delta}(b t, r s, b f, a f)$ \\
\hline \multirow[t]{4}{*}{20} & \multirow[t]{4}{*}{$\overline{b t: b f: a f}$} & $a$ & $\delta(b t, r s, b f, a f)$ & $\overline{\delta(b t, a f)}$ & $\delta(b t, r s, b f, a f)$ & $\overline{b f}$ & $\delta(b t, b f, l s, a f)$ \\
\hline & & $b$ & $\overline{D_{d}}$ & $a f$ & $\delta(b t, r s, b f, a f)$ & $b f$ & $\delta(b f, l s, a f)$ \\
\hline & & $c$ & $\delta(b t, b f, l s, a f)$ & $b f$ & $\delta(b t, b f, l s, a f)$ & $\delta(b t, a f)$ & $\delta(b t, r s, b f, a f)$ \\
\hline & & $d$ & $D_{d}$ & $b f$ & $\delta(b t, b f, l s, a f)$ & $a f$ & $\delta(r s, b f, a f)$ \\
\hline \multirow[t]{5}{*}{21} & \multirow[t]{5}{*}{$b t: l s: a f$} & 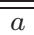 & $\overline{\overline{D_{d}}}$ & $\overline{c \delta(b f, l s)}$ & 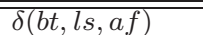 & $\overline{\overline{\delta(r s, a f)}}$ & 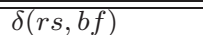 \\
\hline & & $b$ & $\delta(b t, b f, l s, a f)$ & $\delta(b f, l s)$ & $\delta(b t, l s, a f)$ & $\delta(b t, r s, a f)$ & $\delta(b t, r s, b f)$ \\
\hline & & $c$ & $\delta(b t, b f, l s, a f)$ & ls & $\delta(b t, r s, l s, a f)$ & $\delta(b t, r s)$ & $\delta(b t, r s, b f, l s)$ \\
\hline & & $d$ & $\delta(b t, b f, l s, a f)$ & $b f$ & $\delta(b t, b f, l s, a f)$ & $\delta(b t, a f)$ & $\delta(b t, r s, b f, a f)$ \\
\hline & & $e$ & $D_{d}$ & ls & $\delta(b t, r s, l s, a f)$ & rs & $\delta(r s, b f, l s)$ \\
\hline 22 & $r s: b f: l s$ & $\bar{a}$ & $\delta(b t, r s, b f, l s)$ & rs & $\overline{\delta(r s, b f, l s)}$ & $\overline{l s}$ & $\delta(b t, r s, l s, a f)$ \\
\hline \multirow[t]{2}{*}{$\overline{23}$} & \multirow[t]{2}{*}{ rs:bf:af } & $\bar{a}$ & $\bar{\delta}(b t, r s, b f, a f)$ & $\overline{\delta(b t, a f)}$ & $\bar{\delta}(b t, r s, b f, a f)$ & $\overline{\overline{b f}}$ & $\bar{\delta}(b t, b f, l s, a f)$ \\
\hline & & $b$ & $D_{d}$ & $a f$ & $\delta(b t, r s, b f, a f)$ & $b f$ & $\delta(b f, l s, a f)$ \\
\hline 24 & rs:ls:af & $\bar{a}$ & $D_{d}$ & $\overline{l l s}$ & $\bar{c} \delta(b t, r s, l s, a f)$ & rss & $\bar{\delta}(r s, b f, l s)$ \\
\hline \multirow[t]{2}{*}{25} & \multirow[t]{2}{*}{$\overline{\overline{b f}: l s: a f}$} & $\bar{a}$ & $\bar{\delta}(b t, b f, l s, a f)$ & $\overline{b f}$ & $\bar{\delta}(b t, b f, l s, a f)$ & $\overline{\overline{\delta(b t, a f)}}$ & $\bar{\delta}(b t, r s, b f, a f)$ \\
\hline & & $b$ & $D_{d}$ & $b f$ & $\delta(b t, b f, l s, a f)$ & $a f$ & $\delta(r s, b f, a f)$ \\
\hline 26 & $b t: r s: b f: l s$ & $a$ & $I M P$ & $I M P$ & $I M P$ & $I M P$ & $I M P$ \\
\hline \multirow{2}{*}{$\overline{277}$} & \multirow{2}{*}{$\overline{\overline{c b t}: r s: b f: a f}$} & $a$ & $\delta(b t, r s, b f, a f)$ & $\overline{\delta(b t, a f)}$ & $\bar{\delta} \delta(b t, r s, b f, a f)$ & $\overline{\overline{b f}}$ & $\overline{c \delta}(b t, b f, l s, a f)$ \\
\hline & & $b$ & $D_{d}$ & $a f$ & $\delta(b t, r s, b f, a f)$ & $b f$ & $\delta(b f, l s, a f)$ \\
\hline$\overline{228}$ & $\bar{~}$ bt:rs:ls:af & 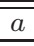 & $D_{d}$ & $\overline{l l s}$ & 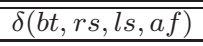 & rss & 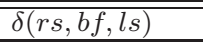 \\
\hline \multirow[t]{2}{*}{29} & \multirow[t]{2}{*}{$\overline{\overline{c b t: b f: l s: a f}}$} & $\bar{a}$ & $\overline{\delta \delta(b t, b f, l s, a f)}$ & $\overline{b f}$ & $\bar{c} \delta(b t, b f, l s, a f)$ & $\overline{\overline{\delta(b t, a f)}}$ & 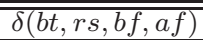 \\
\hline & & $b$ & $D_{d}$ & $b f$ & $\delta(b t, b f, l s, a f)$ & $a f$ & $\delta(r s, b f, a f)$ \\
\hline 30 & rs:bf:ls:af & $a$ & $I M P$ & $I M P$ & $I M P$ & $I M P$ & $I M P$ \\
\hline 31 & $\bar{c} b t: r s: b f: l s: a f$ & $\bar{a}$ & $\overline{I M P}$ & $\overline{I M P}$ & $\overline{I M P}$ & $I M P$ & $\overline{I M P}$ \\
\hline 32 & in & 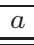 & in & $D_{c}$ & $D_{c}$ & $\overline{\overline{D_{c}}}$ & $D_{c}$ \\
\hline 33 & ou & $a$ & $\overline{\overline{D_{c}}}$ & $\overline{D_{c}}$ & $\overline{\mathrm{Ou}}$ & $\overline{\overline{D_{c}}}$ & $\overline{D_{c}}$ \\
\hline 34 & in:ou & $a$ & $\overline{D_{c}}$ & $D_{c}$ & $D_{c}$ & $\overline{D_{c}}$ & $D_{c}$ \\
\hline
\end{tabular}

TABLE VI

COMPoSING A SINGLE-TILE WITH A BASIC RELATION (2/2)

algorithms of the relations on test data. Then, we checked the results of the experiments to verify that the complete composition table that is found by the program corresponds to the table as it can be computed with algebraic rules.

\section{REFERENCES}

[1] A. Cohn and S. Hazarika, "Qualitative spatial representation and reasoning: An overview," Fundamenta Informaticae, vol. 16, no. (1-2), pp. $1-29,2001$.

[2] M. Egenhofer and J. Herring, "Categorizing binary topological relationships between regions, lines, and points in geographic databases," Department of Surveying Engineering, University of Maine, Orono, ME., 1991.

[3] M. Egenhofer, "Deriving the Composition of Binary Topological Relations," Journal of Visual Languages and Computing, vol. 5, no. 1, pp. 133-149, 1994.

[4] E. Clementini and P. Di Felice, "Spatial operators," SIGMOD Record, vol. 29, no. 3, pp. 31-38, 2000.

[5] D. Waller, J. Loomis, R. Golledge, and A. Beall, "Place learning in humans: The role of distance and direction information," Spatial Cognition and Computation, vol. 2, pp. 333-354, 2000.

[6] E. Clementini, P. Di Felice, and D. Hernández, "Qualitative Representation of Positional Information," Artificial Intelligence, vol. 95, pp. 317356, 1997.

[7] E. Clementini and P. Di Felice, "A global framework for qualitative shape description," GeoInformatica, vol. 1, no. 1, pp. 1-17, 1997.
[8] R. Billen and E. Clementini, "Semantics of collinearity among regions," in OTM Workshops 2005 - 1st Int. Workshop on Semantic-Based Geographical Information Systems (SeBGIS'05), ser. LNCS, R. Meersman, Ed., vol. 3762. Springer-Verlag, 2005, pp. 1066-1076.

[9] E. Clementini and R. Billen, "Modeling and computing ternary projective relations between regions," IEEE Transactions on Knowledge and Data Engineering, vol. 18, no. 6, pp. 799-814, June 2006.

[10] R. Billen and E. Clementini, "A model for ternary projective relations between regions," in Proceedings of EDBT'04, ser. LNCS, E. Bertino, Ed., vol. 2992. Springer-Verlag, 2004, pp. 310-328.

[11] — , "Introducing a reasoning system based on ternary projective relations," in Proceedings of SDH'04, P. Fisher, Ed. Springer-Verlag, 2004, pp. 381-394.

[12] C. Freksa, "Using Orientation Information for Qualitative Spatial Reasoning," in Theories and Models of Spatio-Temporal Reasoning in Geographic Space, ser. LNCS, vol. 639, 1992, pp. 162-178.

[13] A. Scivos and B. Nebel, "Double-Crossing: Decidability and Computational Complexity of a Qualitative Calculus for Navigation," in Spatial Information Theory. Foundations of Geographic Information Science: International Conference, COSIT 2001, ser. LNCS, vol. 2205. SpringerVerlag, 2001, pp. 431-446.

[14] A. Isli, "Combining Cardinal Direction Relations and other Orientation Relations in QSR," in Eighth International Symposium on Artificial Intelligence and Mathematics AI\&M 14-'04, 2004.

[15] G. Retz-Schmidt, "Various views on spatial prepositions," AI Magazine, vol. 9, no. 2, pp. 95-105, 1988.

[16] D. Hernández, Qualitative Representation of Spatial Knowledge, ser. LNAI. Springer-Verlag, 1994, vol. 804.

[17] K.-P. Gapp, "From vision to language: A cognitive approach to the 


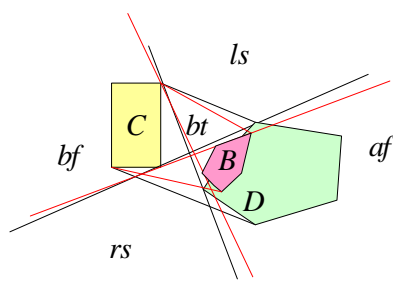

(a)

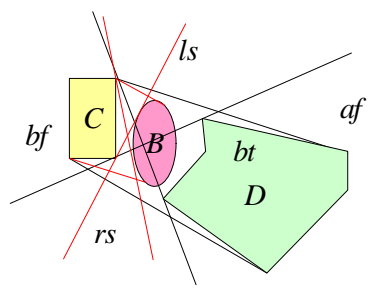

(b)

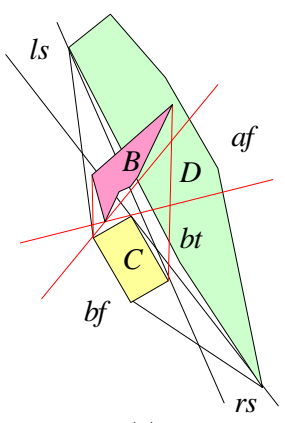

(c)

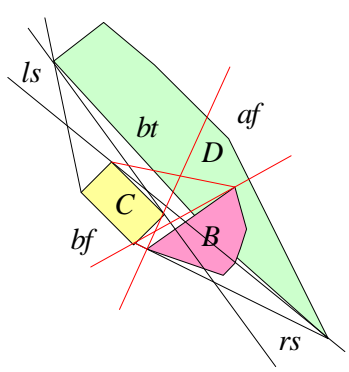

(d)

Fig. 13. Subrows of row $1(b t)$

computation of spatial relations in 3d space," in Proceedings of the European Conference on Cognitive Science in Industry, 1994, pp. 339357.

[18] I. Bloch, O. Colliot, and J. Cesar, R.M., "On the ternary spatial relation "between"," IEEE Transactions on Systems, Man, and Cybernetics, vol. 36, no. 2, pp. 312-327, April 2006.

[19] M. Worboys, M. Duckham, and L. Kulik, "Commonsense notions of proximity and direction in environmental space," Spatial Cognition and Computation, vol. 4, no. 4, pp. 285-312, 2004.

[20] D. Hernández, "Maintaining qualitative spatial knowledge," in Proceedings of COSIT'93, ser. LNCS, A. Frank and I. Campari, Eds., vol. 716. Springer-Verlag, 1993, pp. 36-53.

[21] K.-P. Gapp, "Angle, distance, shape, and their relationship to projective relations," in Proceedings of the 17th Conference of the Cognitive Science Society. Lawrence Erlbaum, 1995, pp. 112-117.

[22] C. Schlieder, "Reasoning about ordering," in Spatial Information Theory: A Theoretical Basis for GIS - Int. Conf., COSIT'95, ser. LNCS, A. Frank and W. Kuhn, Eds., vol. 988. Springer-Verlag, 1995, pp. 341-349.

[23] C. Kray and A. Blocher, "Modeling the basic meanings of path relations," in Proceeding of IJCAI'99. Morgan Kaufmann, 1999, pp. 384-389.

[24] R. Moratz and K. Fischer, "Cognitively adequate modelling of spatial reference in human-robot interaction," in Proceedings of ICTAI 2000, 2000, pp. 222-228.

[25] A. Frank, "Qualitative Spatial Reasoning about Distances and Directions in Geographic Space," Journal of Visual Languages and Computing, vol. 3, pp. 343-371, 1992.

[26] L. Kulik and A. Klippel, "Reasoning about cardinal directions using grids as qualitative geographic coordinates," in Spatial Information Theory. Cognitive and Computational Foundations of Geographic Information Science: Int. Conf. COSIT'99, ser. LNCS, C. Freksa and D. Mark, Eds., vol. 1661. Springer-Verlag, 1999, pp. 205-220.

[27] R. Goyal and M. Egenhofer, "The Direction-Relation Matrix: A Representation for Directions Relations Between Extended Spatial Objects," in UCGIS Annual Assembly and Summer Retreat, June 1997.

[28] C. Vorwerg, G. Socher, T. Fuhr, G. Sagerer, and G. Rickheit, "Projective relations for $3 \mathrm{~d}$ space: Computational model, application, and psychological evaluation," in Proceedings of AAAI'97. AAAI Press / The MIT Press, 1997, pp. 159-164.

[29] H. Schmidtke, "The house is north of the river: Relative localization of extended objects," in Spatial Information Theory. Foundations of Geographic Information Science: International Conference, COSIT 2001, ser. LNCS, D. Montello, Ed., vol. 2205. Springer-Verlag, 2001, pp. 415-430.

[30] L. Kulik, C. Eschenbach, C. Habel, and H. Schmidtke, "A graded approach to directions between extended objects," in Proceedings of GIScience'02, ser. LNCS, M. Egenhofer and D. Mark, Eds., vol. 2478. Springer-Verlag, 2002, pp. 119-131.

[31] S. Skiadopoulos, N. Sarkas, T. Sellis, and M. Koubarakis, "A Family of Directional Relation Models for Extended Objects," IEEE Transactions on Knowledge and Data Engineering, vol. 19, no. 8, pp. 1116-1130, 2007.

[32] V. Dugat, P. Gambarotto, and Y. Larvor, "Qualitative theory of shape and orientation," in Proceedings of IJCAI'99. Morgan Kaufmann, 1999, pp. 45-53.

[33] S. Skiadopoulos and M. Koubarakis, "Composing Cardinal Direction Relations," Artificial Intelligence, vol. 152, no. 2, pp. 143-171, 2004.
[34] - "On the Consistency of Cardinal Directions Constraints," Artificial Intelligence, vol. 163, no. 1, pp. 91-135, 2005.

[35] R. Moratz and M. Ragni, "Qualitative spatial reasoning about relative point position," Journal of Visual Languages and Computing, vol. 19, pp. 75-98, 2008.

[36] C. Gallistel, "Language and spatial frames of reference in mind and brain," Trends in Cognitive Sciences, vol. 6, pp. 321-322, 2002.

[37] F. Tarquini, G. De Felice, P. Fogliaroni, and E. Clementini, "A qualitative model for visibility relations," in Proceedings of KI'07), ser. LNAI, J. Hertzberg, M. Beetz, and E. R., Eds., vol. 4667. Springer-Verlag, 2007, pp. 510-513.

[38] E. Clementini, "Projective relations on the sphere," in 2nd International Workshop on Semantic and Conceptual Issues in GIS (SeCoGIS 2008), ser. LNCS, I.-Y. Song, Ed., vol. 5232. Springer-Verlag, 2008, pp. 313322.

[39] B. Bennett, A. Isli, and A. Cohn, "When does a Composition Table Provide a Complete and Tractable Proof Procedure for a Relational Constraint Language?" in Proceedings of the IJCAI'97 workshop on Spatial and Temporal Reasoning, Nagoya, Japan, 1997.

[40] G. Ligozat, "When Tables Tell it All: Qualitative Spatial and Temporal Reasoning Based on Linear Ordering,' in Proceedings of COSIT'01, ser. LNCS, D. Montello, Ed., vol. 2205. Springer-Verlag, 2001, pp. 60-75.

[41] A. Isli and A. Cohn, "An Algebra for Cyclic Ordering of 2D Orientations," in Proceedings of AAAI'98, July 1998, pp. 643-649.

[42] I. Düntsch, H. Wang, and S. McCloskey, "Relation Algebras in Qualitative Spatial Reasoning," Fundamenta Informaticae, vol. 39, pp. 229-248, 1999.

[43] _ - "A Relation-Algebraic Approach to the Region Connection Calculus," Theoretical Computer Science, vol. 255, pp. 63-83, 2001.

[44] J. Renz and G. Ligozat, "Weak Composition for Qualitative Spatial and Temporal Reasoning," in Proceedings of CP'05, 2005. 